\title{
Stochastic Simulation of Nonequilibrium Heat Conduction in Extended Molecule Junctions
}

\author{
Inon Sharony, ${ }^{\dagger}$ Renai Chen, ${ }^{\ddagger}$ and Abraham Nitzan*, ${ }^{*}, \ddagger$ \\ $\dagger$ School of Chemistry, Tel Aviv University, Tel Aviv 69978, Israel \\ $\ddagger$ Department of Chemistry, University of Pennsylvania, Philadelphia, PA 19104, USA \\ E-mail: anitzan@sas.upenn.edu
}

\begin{abstract}
Understanding phononic heat transport processes in molecular junctions is a central issue in the developing field of nanoscale heat conduction and manipulation. Here we present a Stochastic Nonequlibrium Molecular Dynamics simulation framework to investigate heat transport processes in molecular junctions in and beyond the linear response regime. We use extended molecular models which filter Markovian heat reservoirs through an intermediate substrate region, to provide a realistic and controllable effective bath spectral density. The results obtained for alkanedithol molecules connecting gold substrates agree with previous nonequilibrium Green's function calculations in frequency domain, and match recent experimental measurements (e.g. thermal conductance around $20 \mathrm{pW} / \mathrm{K}$ for alkanedithiols in single molecular junctions) Classical MD simulations using the full molecular forcefield and quantum Landauer-type calculations based on the harmonic part of the same forcefield are compared, and the similarity of the results indicate that heat transport is dominated by modes in the lower frequency range. Heat conductance simulations on polyynes of different lengths illuminates the effects of molecular conjugation on thermal transport.
\end{abstract}




\section{Introduction}

Heat conduction in molecular junctions ${ }^{1}$ has become a subject of increasing interests as a sub-field of nanoscale energy dissipation and transport over the last decade ${ }^{2}$ driven by technological considerations of both stability and functionality of envisioned molecular electronic devices as well as the need to understand the fundamentals of heat transport in nanosize systems. ${ }^{3,4}$ The most common approach to such calculations is based on classical MD simulations with substrate temperature controlled by generalized Langevin baths, with the obvious deficiency of misrepresenting the dynamics of high frequency modes, relying on the assumption that molecular heat conduction is dominated by modes in the lower frequency regime.

Alternatively, quantum calculations were done, mostly based on the non-equilibrium Green's Function (NEGF) ${ }^{5,6}$ methodology usually using the harmonic part of the molecular force field, leading to Landauer-type expressions ${ }^{7}$ for the molecular heat conduction in the harmonic approximation analogous to its counterpart in the problem of molecular electronic transport using free electron models. Given the different ranges of applicability of classical dynamics on the one hand and harmonic quantum dynamics on the other, comparing their performance in evaluating and predicting heat molecular conduction is obviously of interest.

Another powerful tool to investigate many-body interactions, without necessitating the harmonic approximation, is using atomistic Molecular Dynamics (MD) simulations. MD simulation of an ergodic system allows calculation of Statistical Mechanical properties of a system (e.g. thermal conductivity) by analysis of the atomic trajectories. MD simulations from previous reports, however, are mostly focused on specific systems such as liquids, ${ }^{8}$ thin-

films,${ }^{9}$ Graphene, ${ }^{10}$ or one-dimensional metal/semi-metal chains or wires. ${ }^{11}$ It is unclear how applicable these system-tailored simulations are to the thermal conduction in Single Molecule Junctions (SMJ). A fully-functional MD simulation tool to study the structural dependence of molecular heat conduction, in which a full force-field is applied without particular system restrictions, is still lacking.

Focusing on classical simulations, equilibrium MD (EMD) is one of the easiest approaches 
to implement. One essentially applies the Green-Kubo formula to the time-autocorrelation of the current to get the thermal conductivity in the linear response regime. ${ }^{12-19}$ Besides its limitation to linear response approximation, the method also suffers from slow convergence and limited applicability to heterogeneous systems. ${ }^{15}$ Alternatively, under the nonequilibrium MD (NEMD) approach ${ }^{10,15,20-23}$ one creates a temperature gradient by separating the simulated system into "slabs", and rescaling the atomic velocities at the "heat source" and âĂIJsinkâĂİ slabs to set the temperature boundary conditions. In implementing this methodology care has to be taken for the finite-size effects associated with the so-imposed boundary conditions. ${ }^{15,19}$ Plus, the thermal bath effects are relatively obscure for this method. Another popular tool is the so-called Reversed Nonequilibrium MD (RNEMD) $)^{8,24-30}$ in which the effect (fluxes) and the cause (temperatures) are reversed: one creates temperature differences by separating the simulated system into "slabs", and enforces a given heat flux on the system by taking a certain amount of kinetic energy from the "heat source" slab and put it into the "heat sink" slab, until the system reaches steady state at which the temperature at the source and sink sides is determined. Since in this approach non-equilibrium is imposed by a constant heat flux, it is limited to steady-state calculations.

The stochastic nonequilibrium MD (SNEMD) methodology used in the present work is a variant of the NEMD outlined above, in which velocity rescaling reflects the interaction with a generic (white) thermal bath in a way consistent with the fluctuation-dissipation theorem. To account substrate actual spectral properties a section adjacent to the molecule is modeled explicitly and filters the effects of the generic stochastic dynamics ${ }^{31}$ applied to bulk layers further from the molecular bridge. We show the stability and applicability of our method in calculating the temperature distribution, heat current, and thermal conductance in various molecular junction settings. Under our approach, the concept of temperature and build-up of thermal bias come in naturally, without manually perturbing the system at each simulated time-step or reversing causality.

This paper is the first in a series in which we plan to study the interplay between molecular 
composition and structure and its heat transport properties. For this purpose we have developed a numerical tool (described below) that can be readily adapted to different molecules and structures. Here we apply our tool to the study of heat transport in single alkane chains, a system that has been studied numerically ${ }^{32,33}$ and experimentally (mostly for alkane layers ${ }^{34-36}$ but very recently, for the first time, also for single alkane chains ${ }^{37}$ ). Our results serve to test our calculation against previous calculation and most importantly against recent experimental results as well as Landauer based harmonic quantum calculations, and demonstrate the applicability robustness of these calculations. Furthermore, we present heat conduction results also for a series of conjugated carbon chains âĂŞ- better candidates for molecular electronic transport applications ${ }^{38,39}$ but, as be find, similar to their saturated counterparts in their heat transport behavior.

Section 2 provides details on our simulation technique and our code. Section 3 discuss the results of heat conduction properties of different types of hydrocarbon chains within molecualr junctions using such approach, and compare them to the existing theoreical and experimental data. In Section 4 we conclude and give future directions of research in this series.

\section{Model and Calculations}

While the energy spectrum of molecular vibrations encompass a relatively large ( 0 âĂŞ 0.5 $\mathrm{eV}$ ) frequency range, high-frequency vibrations tend to be spatially localized and energetically above the cutoff frequency of many solid-state substrates. For these reasons, and also because such modes are not populated at room temperature, they contribute little to molecu-

lar heat transport at that temperature. ${ }^{32}$ Molecular heat transport is therefore dominated by lower frequency vibrations, for which classical dynamics provide a reasonable approximation. Molecular Force-Fields (FF) allow efficient representation of a classical, anharmonic molecular Potential Energy Surface (PES) which is the input to the SNEMD studies described 
below.

In the present work we chose to represent the attributes of the thermal environment using an explicit thermal bath. As shown in figure 2, we extend our molecular system with one or more atomic layers of the substrate, while the bulk atoms furthest from the molecular system are subjected to Markovian white noise which is thus filtered by the explicit substrate layers. Specifically, the interfaces between the molecule and the baths (Region III) on either side of it are denoted as Region II in the diagram (Figure 1). They are modeled using the same Molecular Mechanical Force Fields as the molecular system, which are optimized for small organic and organometallic molecules (more details later in this section). The interfaces are comprised of an explicit part of the bulk, which can be seen as the tips of the measuring apparatus, and are usually composed of layers of metallic materials (e.g gold, platinum).

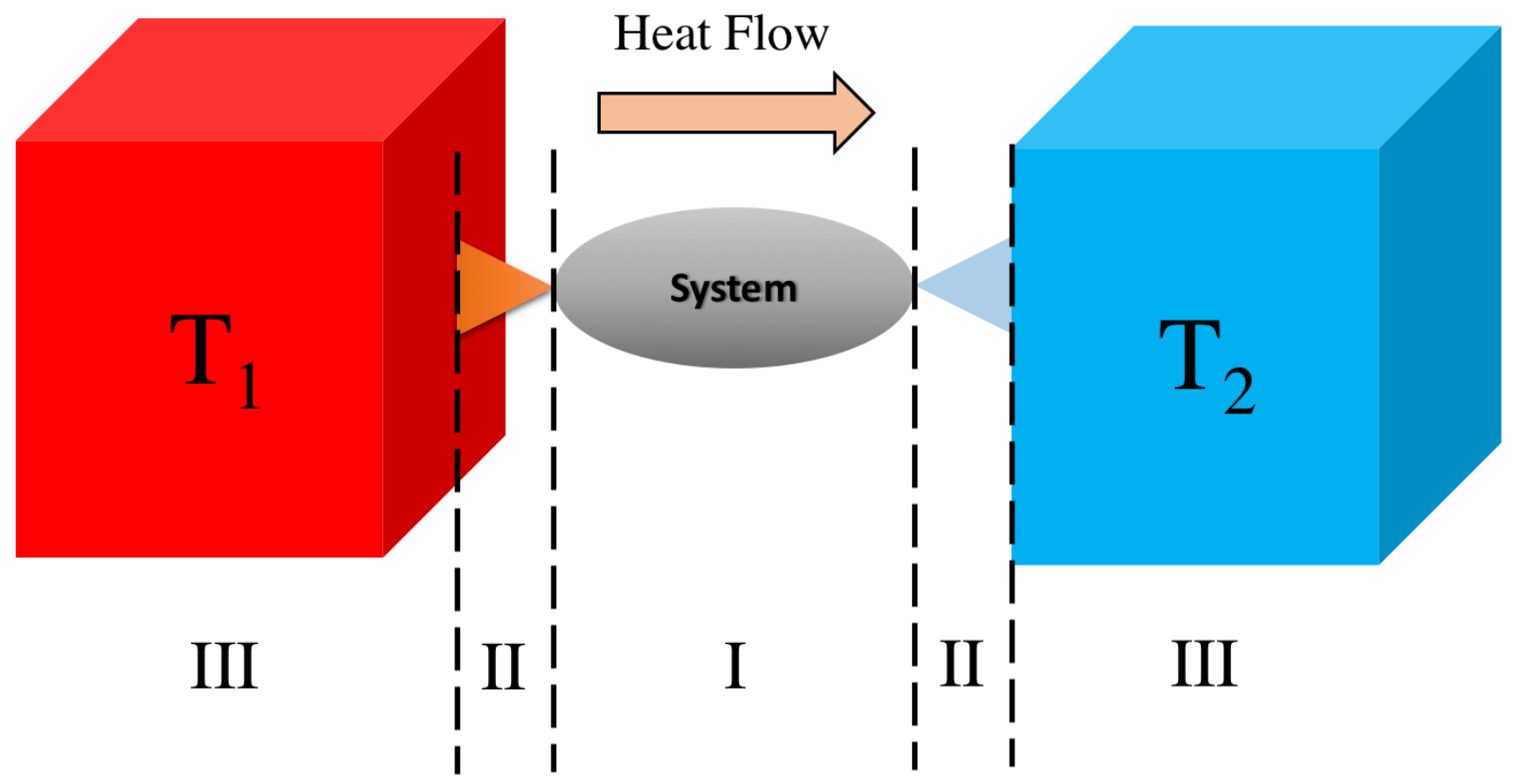

Figure 1: A schematic diagram of the explicit bath model. Region I is the molecular system (including thiol groups); Region II represents the interface and is comprised of explicit layers of metallic materials; Region III are implicit baths representing the infinitely large thermal reservoirs, exerting white noise.

The calculation of the heat current starts by representing the potential energy of the whole system as a sum of individual interaction terms $V_{\tau}$, where $\tau$ refers to different interac- 
tion types, for example, the two-body interaction between atom 1 and 2 or the three-body interaction between atoms 1,2 , and 3 . We assume an interaction term $V_{\tau}$ can be further separated as a weighted sum over the atoms connected by it, weighted according to some partition scheme. A generalized analytic formalism has been explored by Torii et al., ${ }^{40}$ explicitly

$$
\begin{aligned}
E_{\text {tot }} & =\sum_{i}^{N} \frac{1}{2} m_{i} \mathbf{v}_{i}^{2}+\sum_{\tau} V_{\tau} \\
V_{\tau} & =\sum_{j}^{n(\tau)} U_{\tau, j}, \\
U_{\tau, j}\left(\left\{\mathbf{r}_{1} \ldots \mathbf{r}_{n(\tau)}\right\}\right) & =C_{\tau, j} V_{\tau}\left(\left\{\mathbf{r}_{1} \ldots \mathbf{r}_{n(\tau)}\right\}\right), \\
\sum_{j}^{n(\tau)} C_{\tau, j} & =1,
\end{aligned}
$$

where $n(\tau)$ is the number of atoms connected by the interaction $V_{\tau}$. The fraction, $C_{\tau, j}$, of potential energy from $V_{\tau}$ assigned to atom $j$ is termed as $U_{\tau, j}$. Assigning specific energies to individual atoms is necessary in order to define atomic energies and energy flows between atoms, but is obviously somewhat arbitrary. In our modeling we chose to assign equal partitioning of each potential energy term between the individual participating atom. With such partitioning defined, the heat flux associated with a given atom $\mathrm{i}$ in the molecular system, is given by

$$
J_{i} \equiv \frac{d E_{i}}{d t}=\frac{d}{d t}\left(\frac{1}{2} m_{i} \mathbf{v}_{i}^{2}+\sum_{\tau} U_{\tau, i}\right)=\sum_{\tau} \sum_{j=1}^{n(\tau)} J_{\tau, i j}
$$

where the heat flux going from atom $j$ to atom $i$ which are connected by $V_{\tau}$ is defined as,

$$
J_{\tau, i j}=C_{\tau, j} \mathbf{f}_{\tau, i} \cdot \mathbf{v}_{i}-C_{\tau, i} \mathbf{f}_{\tau, j} \cdot \mathbf{v}_{j}
$$


We have defined $\mathbf{f}_{\tau, j}$ as the force derived from interaction $U_{\tau, j}$. This is the core expression we use to calculate the inter-atomic heat currents. In addition to the inter-atomic force fields we add the effect of the thermal baths through damping forces and random fluctuations from Langevin Dynamics. We expect heat current values to plateau as the system tends towards steady-state.

A customized Molecular Dynamics (MD) package built around GROningen MAchine for Chemical Simulations (GROMACS) $4.5^{41}$ is developed and utilized to conduct the simulations. The leap-frog algorithm (provided by GROMACS) is used for propagation of the deterministic parts of the system, while Langevin dynamics are used to propagate the stochastic parts of the simulation. ${ }^{31}$ Unless otherwise stated, the time step is always 1 fs for all runs and the coupling strength between the Markovian bath and outermost layer of explicit bulk (region is 1 ) is $p s^{-1}$.

First, different utilities, are used to prepare the initial conditions for the simulation. These include open source software Open Babel, ${ }^{42}$ Avogadro ${ }^{43}$ and other homemade programs and scripts for creating input topologies and indices. The Universal Force Field (UFF) ${ }^{44}$ parameters are chosen throughout the simulations.

UFF is one of a few force fields that includes most of the atomic types and bonds across the periodic table, and thus is suitable for organometallic junctions. As the high frequency carbon-hydrogen bonds often contribute little to the overall vibrational heat conduction, it is reasonable to compare side-by-side the effect of with and without hydrogen explicitly appear in the force field. This will in principle determine whether it is a good approximation to use unified-atom (ua) version of UFF over all-atom(aa) UFF. 


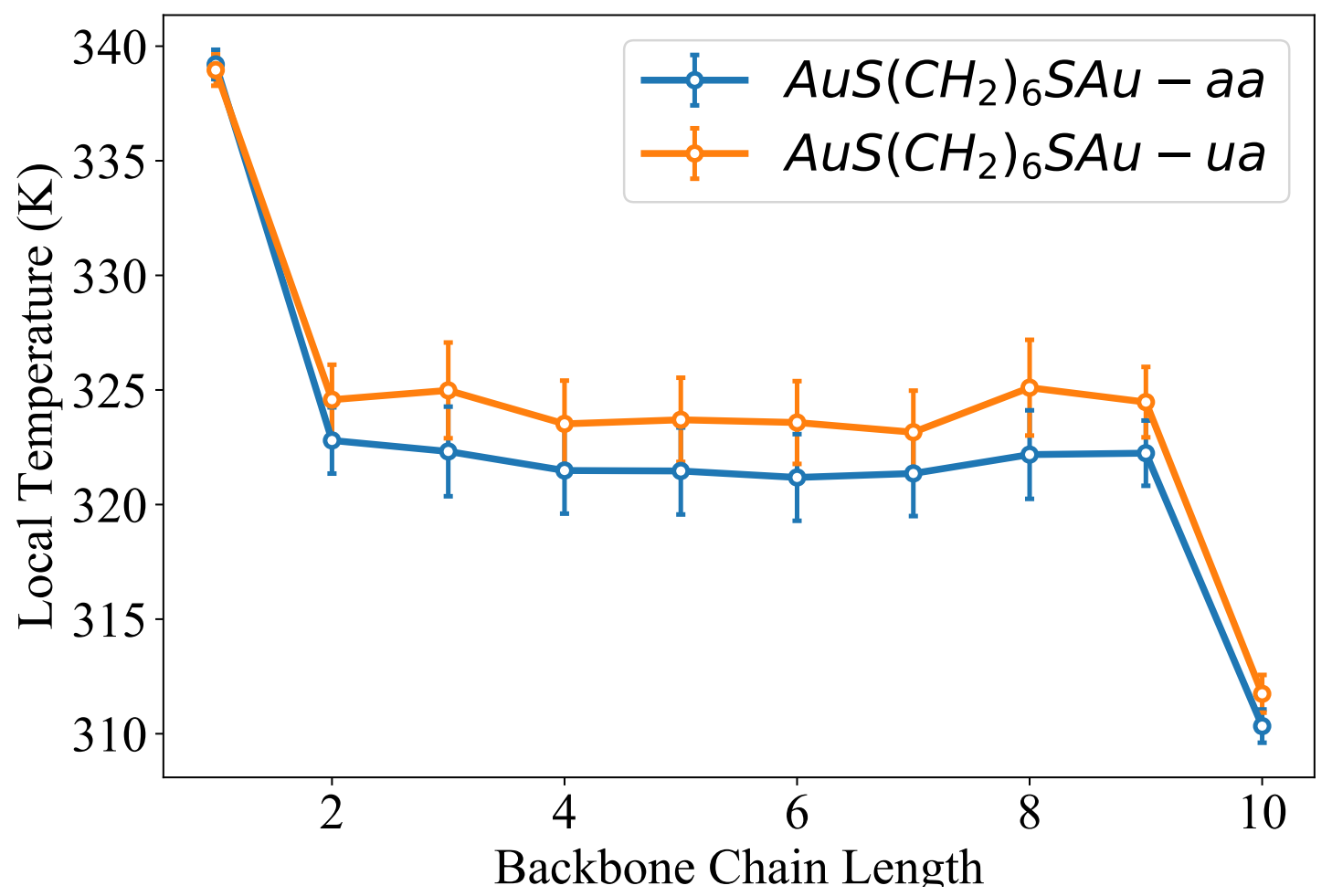

Figure 2: Temperature profile for 1,6-hexanedithiol, comparing UFF all-atom and UFF unified-atom force fields. The horizontal axis is labeled according to the index of the backbone atoms (Sulfur atoms included), and also including the layer of Gold atoms nearest the molecule (the leads), to the left most and right most. The temperatures of the left and right leads are set at $350 \mathrm{~K}$ and $300 \mathrm{~K}$ respectively. The error bars represent the standard error.(see also Caption in Figure 4)

Table 1: Heat conductance difference between UFF all-atom and UFF unified-atom force fields, for 1,6-hexanedithiol with three layers of Gold electrodes on each side. SD stands for standard deviation, and SE for standard error. All values are given in units of pico-Watts per Kelvin.

\begin{tabular}{||cccc||}
\hline & Conductance & SD & SE. \\
\hline All-Atom & 20.73 & 7.44 & 0.74 \\
Unified-Atom & 21.77 & 6.40 & 0.64 \\
\hline
\end{tabular}

The local temperatures of the backbone carbons (together with sulfurs and first layer of gold) are reasonably unchanged when change the UFF-aa to UFF-ua (2). Table 1 compares the effect of force-field choice between all-atom and a unified-atom approximation of UFF, 
on the heat conduction of the hexanedithiold molecule. The relative symmetric difference ${ }^{1}$ between the calculation results for the two force-fields is $4.89 \%$, and Welch's t-test ${ }^{2}$ is $13 \%$. Therefore, we conclude that the unified-atom approximation is acceptable for our purposes.

The simulation begins by preparing the desired molecular state through building of the junction structure, and optimizing its geometry to be at the configuration of minimal energy. The structure is equilibrated to the average temperature of the baths, and then propagated under the boundary conditions of the required temperature bias (e.g. 300K and 350K) until it reaches steady state (usually about a few nanosecond). The steady state trajectories are sampled under this specific temperature bias. Pairwise forces between atoms, and between each bath and the atoms coupled to it, are also sampled.

Finally, the heat currents are calculated from the trajectories and forces. The heat currents are then time-averaged. Ensemble-averages are performed to obtain statistically sound final currents and conductance.

As for Landauer-type calculations, a detailed description of the formalism is provided in the Supporting Information (SI), together with other relevant data and figures.

The heat current equations and computational apparatus described above were used to calculate individual heat currents between any two atomic pair within the molecular systems. This is not limited to nearest-neighbour bonded atoms (bond stretching interactions), but also applies to atoms that are three or four sites apart but still interconnected by other interactions parameterized in the force fields (e.g., angle bending, torsion, etc.) The heat current flowing from one heat bath to the other bath in the molecular junction, can be measured by setting up an imaginary plane which is perpendicular to the longitudinal axis of the molecule and sum over all the inter-atomic heat currents going from one side of the plane to the other side of the plane. In steady-state, the heat current through the molecule will be measured the same, regardless of where we chose to draw this imaginary plane. For

\footnotetext{
${ }^{1}$ The relative symmetric difference is $\delta(x, y)=\frac{|x-y|}{(x+y) / 2}$

${ }^{2}$ Welch's t-test is $\eta(x, y)=\frac{|\mathbf{E}[x]-\mathbf{E}[y]|}{\sqrt{\sigma_{x}^{2}+\sigma_{y}^{2}}}$, where $\sigma_{x}$ is the Standard Deviation in random variable $x$
} 
computational simplicity, we chose to draw it between region I in figure 1 (molecular) and region II (the substrate interface). The average thermal conductance is defined as the ratio between this quantity and the temperature bias between the hot and cold baths,

$$
\kappa=\frac{J_{\text {tot }}}{T_{\text {hot }}-T_{\text {cold }}}
$$

In addition to heat fluxes, the local temperature of each atom in the conducting molecule is calculated from the statistically averaged kinetic energy of the atoms. More details regarding our methodology are given at the end of this article and in the Supporting Information (SI).

\section{Results and discussion}

The system under investigation is a Single Molecule Junction comprising an alkanedithiol $\mathrm{HS}\left(\mathrm{CH}_{2}\right)_{\mathrm{n}} \mathrm{SH}$ as a molecular bridge connecting several layers of explicit bulk atoms. We compared alkanedithiols of various lengths (measured by the number of Carbon atoms in the

alkane backbone), and explicit bulk comprised of one to four layers of gold atoms which are further connected to bulk substrates. Some examples are illustrated in Figure 3. For each molecular species, we performed MD simulations of the non-equilibrium molecular junction, evaluating its steady-state heat transport behavior following the procedure described in Section 2. 
(a)

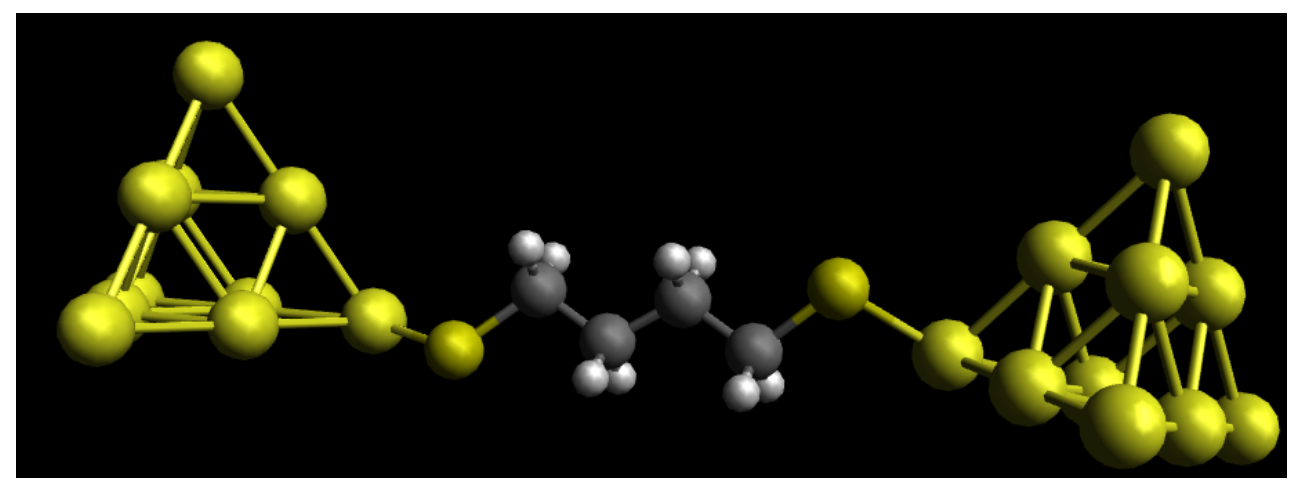

(b)

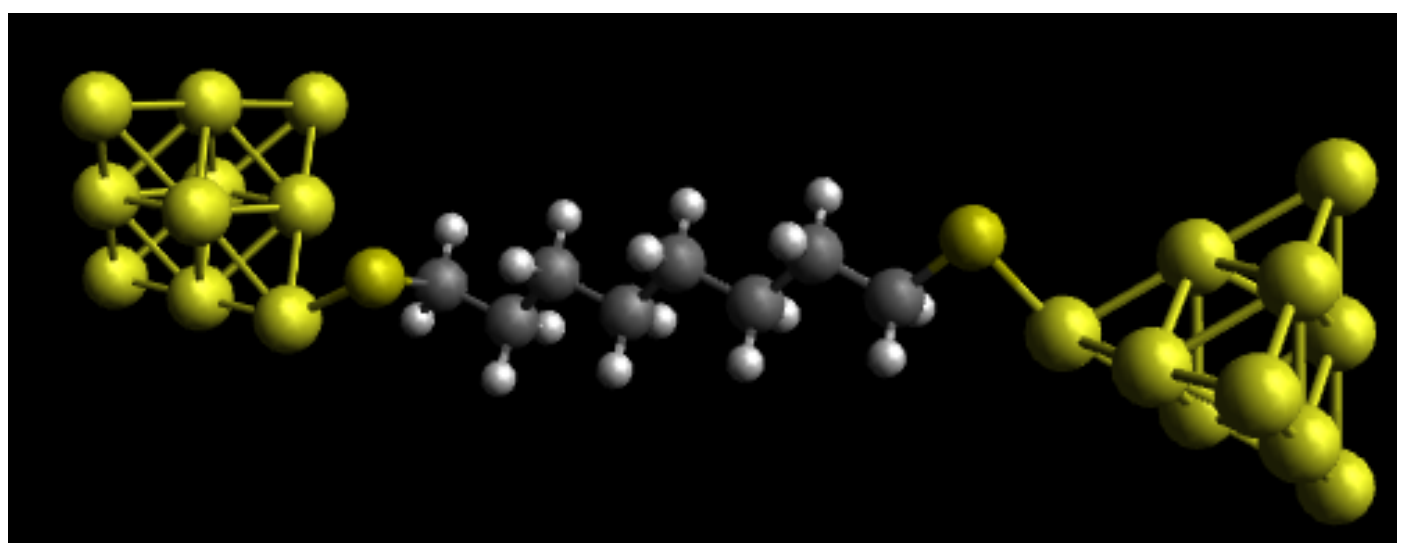

Figure 3: Illustration of some of the alkane molecules studied in the simulation, with three layers of explicit gold bulk. The white noise thermostats are only attached to the layer of gold atoms furthest from the alkane bridge. (a) 1,4-butanedithiol $\left(\mathrm{HS}\left(\mathrm{CH}_{2}\right)_{4} \mathrm{SH}\right)$; (b) 1,8octanedithiol $\left(\mathrm{HS}\left(\mathrm{CH}_{2}\right)_{8} \mathrm{SH}\right)$.

For each molecular species, we performed MD simulations of the molecular junction at Nonequilibrium Steady-State (NESS). More computational details are given in the SI.

In order to ascertain the relevance of the explicit baths modelling, we compared results for molecular heat conductance using different numbers of explicit gold layers to represent the bulk. Specific simulations are performed on hexanedithiol (Figure 4). The calculated conductance appears to converge when three layers of explicit gold are used in the substrate representation. A similar saturation of layer effect was found also in the other hydrocarbon molecules (See SI for results of more alkanedithiols). In agreement with this observation, a study by Zhang et. al. on self-assembled monolayers showed that the effect of the baths on the molecular system is mainly due to the first few layers of gold substrate. ${ }^{45}$ As seen in Fig. 5 , the observed convergence reflects the convergence in spectral density properties of the gold 
clusters used to represent the thermal baths. It should be noted that to enforce the junction geometry, the explicit bulk is position-restrained by a harmonic force acting on the layer of atoms furthest from the molecule. The spectral densities in Fig. 5 are calculated for the position-restrained clusters since the position restraint force is part of the spectral density affecting the molecule. The signature of the harmonic position-restraining force is evident at its frequency of about 40 wavenumbers. The rest of the spectrum shows frequencies in the range of tens to a few hundred wavenumbers, which agrees with experimental measurements of vibrational DOS for gold nanoparticles. ${ }^{46}$

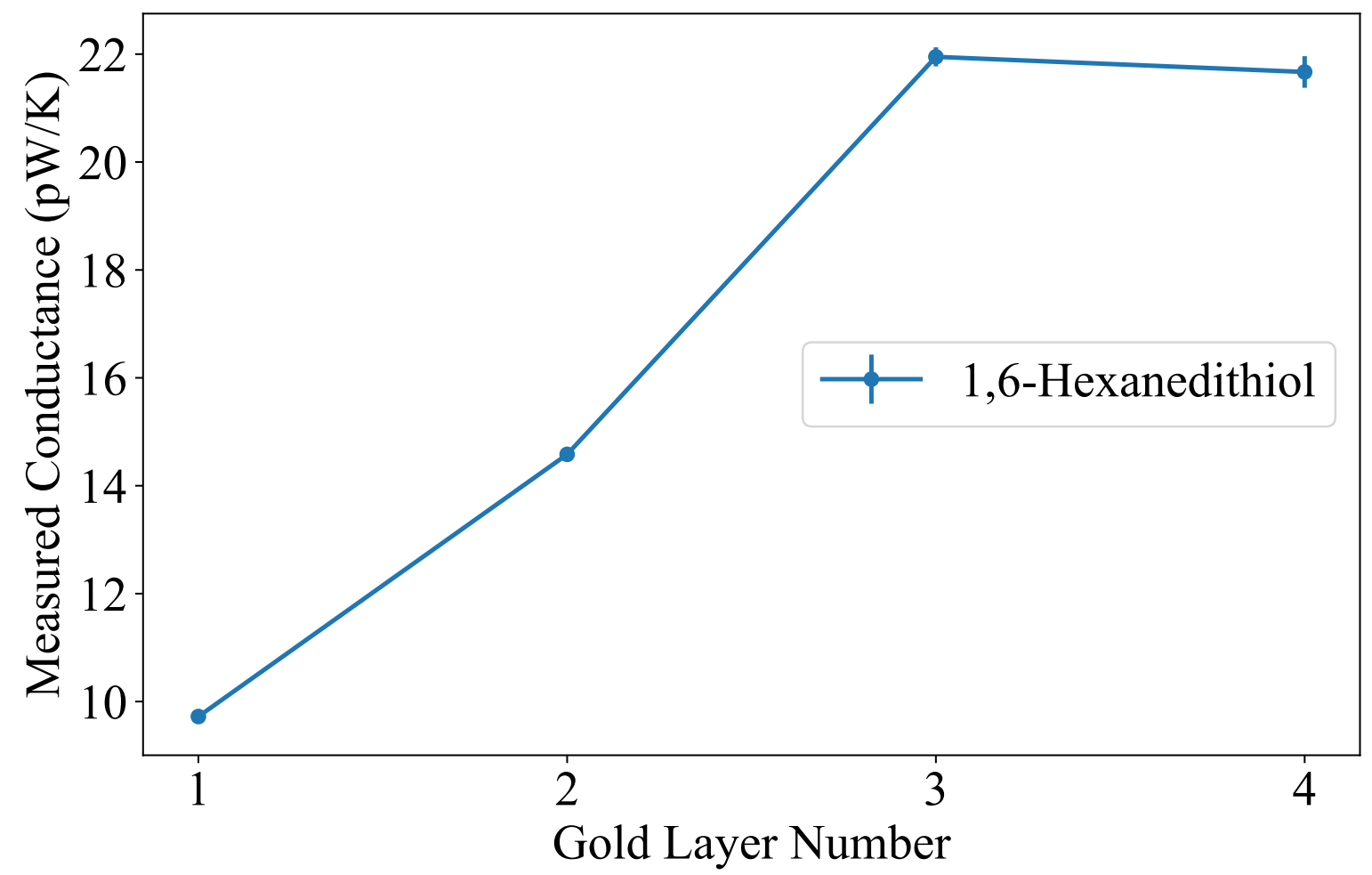

Figure 4: Heat conductance for the molecule $\mathrm{HS}\left(\mathrm{CH}_{2}\right)_{6} \mathrm{SH}$ given different numbers of gold layers as the explicit bulk. The temperature bias is set at $300 \mathrm{~K}$ to $350 \mathrm{~K}$. The bars shown in the figure are the standard errors (SE) of the conductance measurements. ( $\mathrm{SE}=\mathrm{Standard}$ Deviation (SD) / square root of the sample size, is a statistical uncertainty indicator of the estimated mean value of the conducted measurements. ${ }^{47}$ ) 
(a)

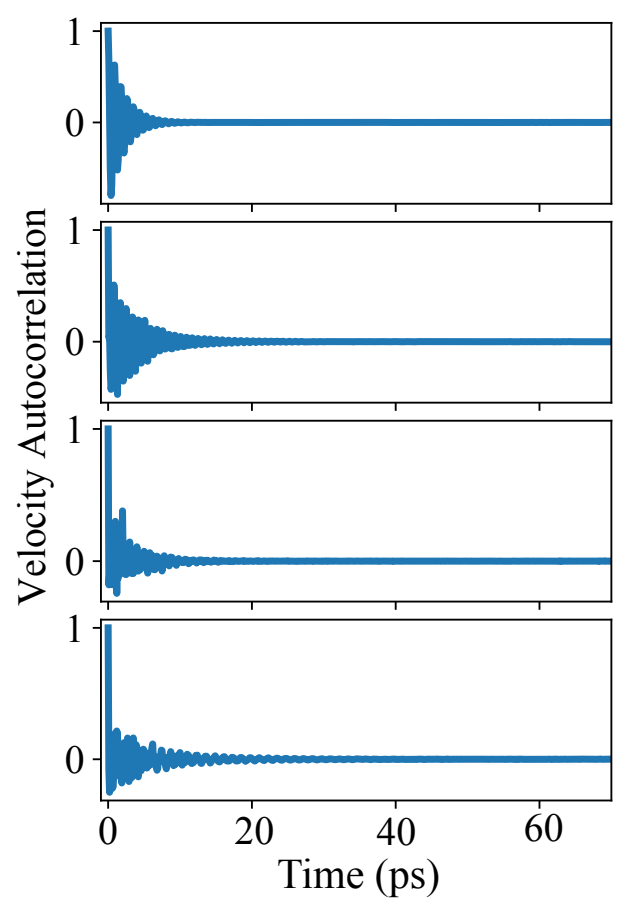

(b)

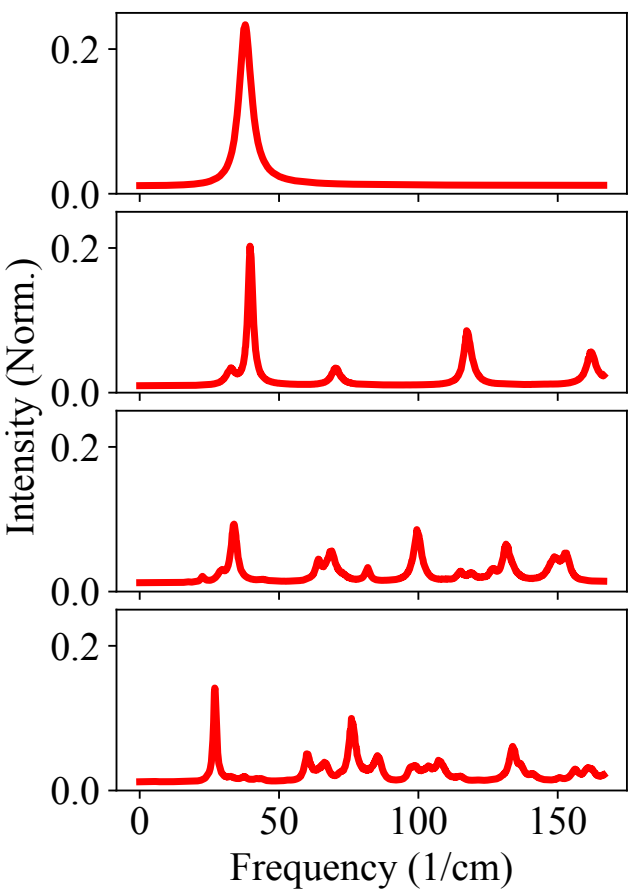

(c)
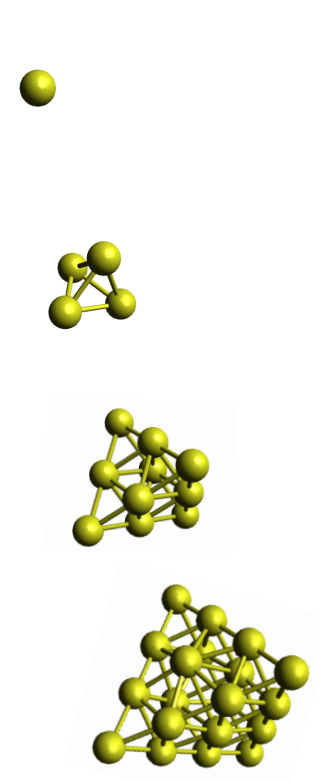

Figure 5: Velocity-velocity autocorrelation functions of the only atom in the first layer of each of four different gold clusters. The outer-most layers are attached to Markovian thermal reservoirs at temperature of $300 \mathrm{~K}$ and the clusters are allowed sufficient time (e.g. a few nanoseconds) to relax to the temperature of the bath. Column (a): Velocity timeautocorrelations, $C_{v v}(t)$, which are normalized to the value at $t=t_{1}-t_{2}=0$; Column (b): The Fourier transforms of the corresponding correlations, normalized across the whole spectra; Column (c): Artistic representation of the corresponding gold clusters

To study the effect of the molecular structure on the junction conductance, we compared molecules of various lengths and of different degrees of saturation. Namely, we compared molecules with an alkane backbone (saturated) against molecules with a conjugated polyyne backbone (unsaturated) ${ }^{3}$. Unless otherwise specified, the simulation results displayed below were obtained using three explicit atomic layers for the gold substrates. Figure 6 shows that heat conductance of shorter molecules $(n<6)$ is not strongly affected by carbon bond saturation, however longer unsaturated chains exhibit lower conductance than their saturated counterparts. This observation stands in contrast to the higher electronic transport properties of conjugated chain molecules, ${ }^{38}$ and may be explained by the difference in current

\footnotetext{
${ }^{3} \mathrm{~A}$ polyyne is an organic compound with alternating single and triple bonds; that is, a series of consecutive alkynes, $(-C \equiv C-)_{n}$ with $n$ greater than 1 .
} 
carriers of thermal and electronic transport in these systems: While the delocalized electrons in conjugated molecules may contribute much to the overall electronic conduction, heat conduction, which is dominated by phonon transport, is mostly determined by bond structure and vibrational modes in the molecular system. The steady state temperature profiles associated with the results of Figure 6 are displayed in Figure 7. The bias (300K - 350K) clearly exceeds the regime of validity of linear response, yet is more realistic with respect to existing experimental setups. ${ }^{34-37}$

The temperature profiles show that most of the thermal resistance is interfacial. Even the longer molecules are homogeneous enough that the temperature profile does not slope significantly, which could point to a ballistic regime of heat conduction in the molecular bridge. This can be ascribed to the explicit modelling of the molecule-bulk interface at the atomic level. The features of interfacial temperature already show some filtering effects from the white baths, noting the first layer of gold bulk on the left is about 10 degrees lower than external hot reservoir and right first layer 10 degrees higher than the external cold reservoir. 


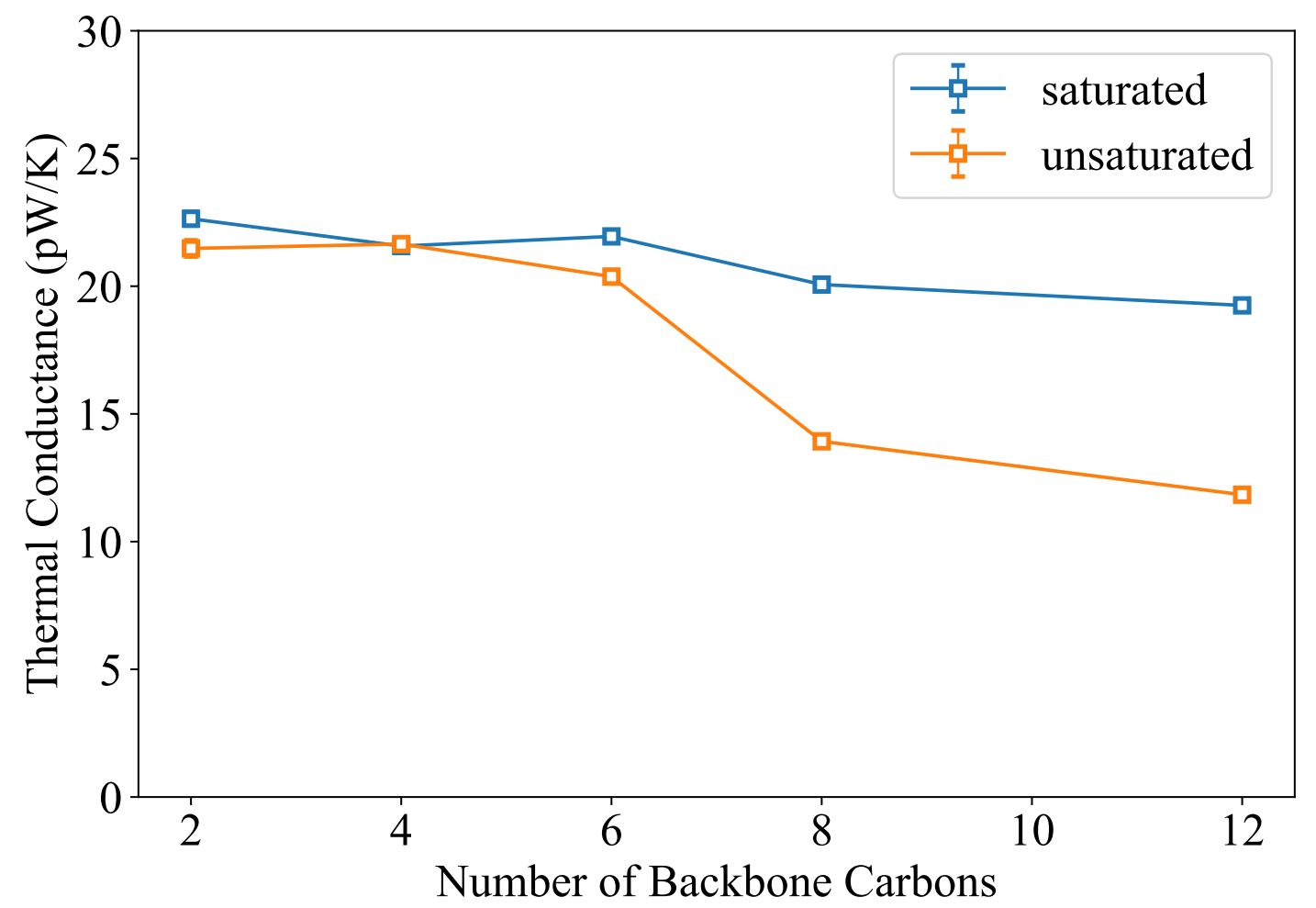

Figure 6: Length-dependent heat conductance of alkane chains (saturated) and triple-bond conjugated hydrocarbon chain (unsaturated) molecules for a temperature bias of $300 \mathrm{~K}$ and $350 \mathrm{~K}$. The error bars represent the SE (see Caption in figure 4) of the conductance measurements. 


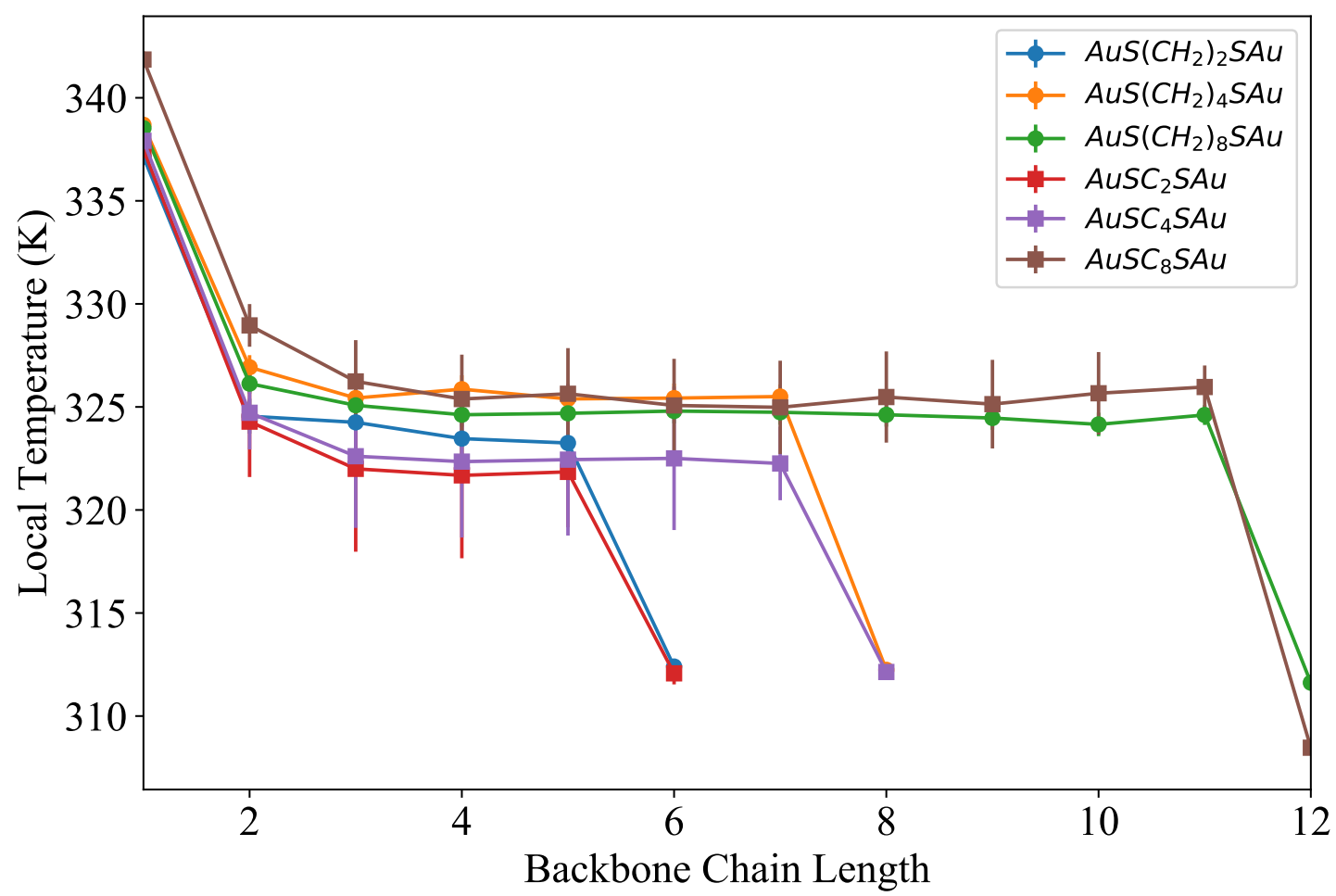

Figure 7: Temperature profile for non-branching alkanedithols of various lengths. In the legend the ones with and without hydrogen atoms are alkanes and conjugated polyyenes respectively. The horizontal axis is labeled according to the index of the backbone atoms (Sulfur atoms included), and also including the layer of gold atoms nearest the molecule (the leads), to the left most and right most. The temperatures of the left and right leads are set at $350 \mathrm{~K}$ and $300 \mathrm{~K}$ respectively. That is, atom zero is always the gold atom to the left of the alkanedithiol, atom one is the left Sulfur atom, and the same on the right. The error bars represent the SE (see Caption in figure 4) the temperature measurements.

Next, consider the results obtained from the quantum-mechanical harmonic model calculation. The theoretical and numerical details of the Landauer approach for calculating heat conductance has been specified in the Support Information. Figure 8 compares the results of this model to those obtained from the classical MD simulation. While a certain degree of divergence occurs of these two methods in the results of alkanedithiolds (which we will explain in the later parts in the section), the remarkable agreement in polyynes molecules indicates that heat conduction in these systems is determined by the harmonic part of the force field and dominated by modes from the lower frequency range of the molecular spectrum. 


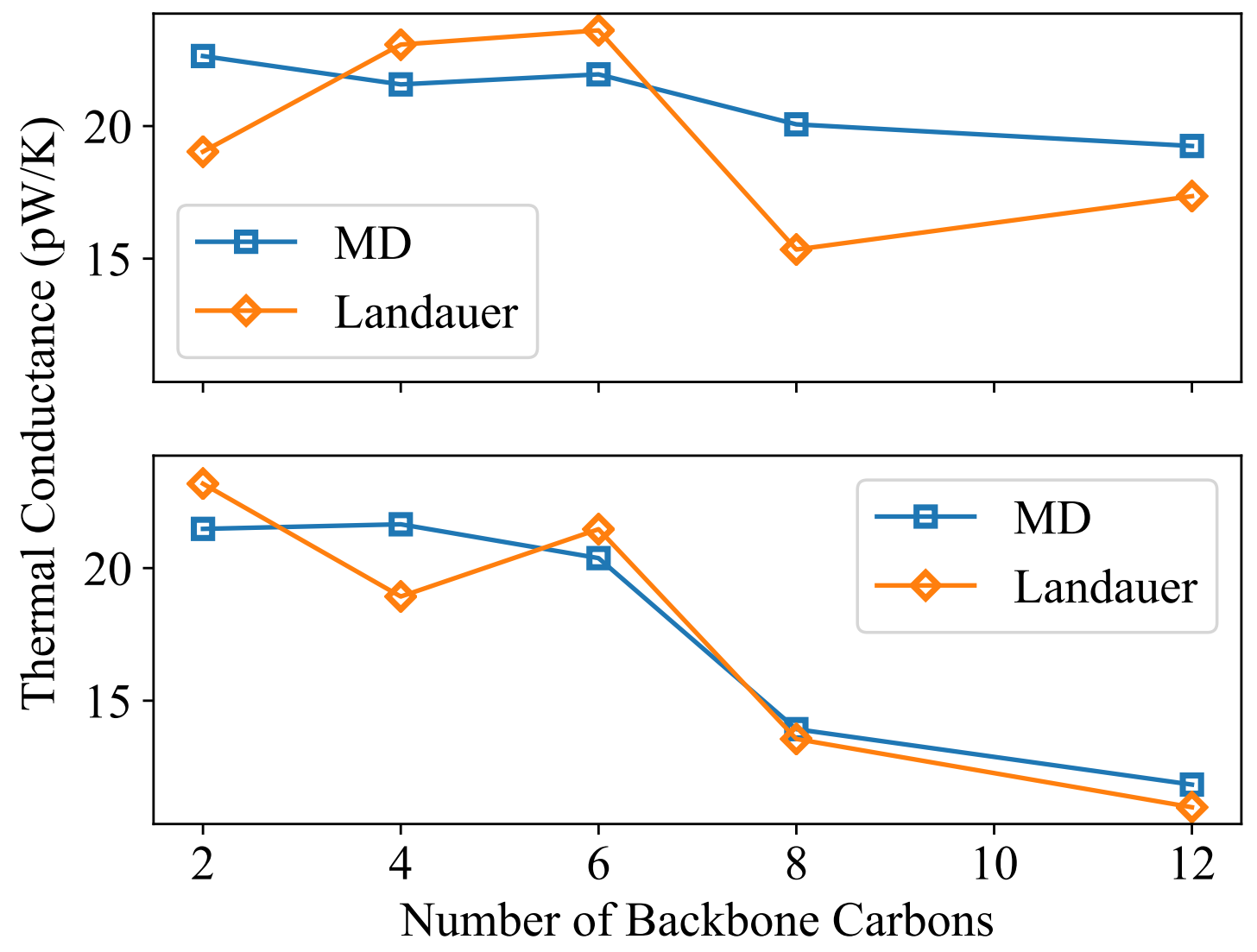

Figure 8: Length-dependent heat conductance of alkane chains (saturated, in the upper panel) and triple-bond conjugated hydrocarbon chain (unsaturated, in the lower panel) molecules, obtained with GROMACS MD simulations and Landauer-type quantum calculations, respectively. In all cases, thiol head-groups are attached, and the currents are calculated across a temperature bias from $350 \mathrm{~K}$ to $300 \mathrm{~K}$, with conductance defined as the ratio between heat current and temperature bias (50K). 
Finally, consider the absolute values of the calculatioted heat conductions. In general, our results are in agreement with the most recent experimental measurements for heat conduction in SMJs. ${ }^{37}$ For the saturated alkanes, the Landauer conductance starts to fluctuate and then goes down as the chain grows even longer (See SI for more data), while the MD results decrease slowly and stay relatively stable around the value $20 \mathrm{pW} / \mathrm{K}$. Though the non-monotonic behavior of the Landauer calculations align with an independent ab initio Landauer-type calculation, ${ }^{33} \mathrm{MD}$ simulations align more closely with the experimental data and trend, ${ }^{37}$ indicating anharmonicity plays a tunning role in molecular thermal transport. It is interesting and intuitive to see heat conduction from a normal mode harmonic perspective (i.e. the normal mode density, localization, and transmission probablity, see SI for

detailed analysis), but at room temperature classical MD seems do better in taking into account both harmonic and anharmonic effects by utlizing full-force-field interactions of the molecular potential energy.

\section{Conclusion}

In summary, we have presented results of classical MD simulations using stochastic Langevin thermal baths, as well as results of a quantum calculation based on the harmonic part of the molecular force field, for the steady-state heat conduction of molecular junctions comprising saturated and conjugated hydrocarbon chains connecting gold leads. The multiple layers of explict gold substrates act as filters of larger environmental white noise and bring characteristic bath effects to the heat conducting molecular systems under investigation. The high degree of agreement between our simulations and the most recent experimental measurements $^{37}$ also validates the methods and numerical tools we use. For the alkanedithiols in particular, MD simulation agrees better with the experiment than Landauer-type quantum calculation in conditions of room temperature and with large bias. This may hint that at ambient conditions, the explicit treatment of quantum effects is less relevant than 
explicit treatment of anharmonicity, and linear response less relevant than the behavior of systems far-from-equilibrium. For conjugated hydrocarbons, while previous studies have shown unusually high electronic conduction of polyynes, ${ }^{38}$ our study shows that they have a lower thermal conductance than their saturated counterparts. which might indicate they are potentially good candidates for thermoelectric nanomaterials. Still the effects of complex molecular structures and topologies on nanoscale heat conduction are still not clear, and will be future directions of our research in the group by making full use of the atomistic-resolution afforded by our approach.

\section{Acknowledgement}

The research of AN is supported by the Israel-U. S. Binational Science Foundation, the German Research Foundation (DFG TH 820/11-1), the U. S. National ScienceFoundation(GrantNo. CHE1665291), and the University of Pennsylvania.

\section{References}

(1) Segal, D.; Agarwalla, B. K. Vibrational Heat Transport in Molecular Junctions. Annu. Rev. Phys. Chem. 2016, 67, 185-209.

(2) Pop, E. Energy dissipation and transport in nanoscale devices. Nano Res. 2010, 3, $147-169$.

(3) Li, N.; Ren, J.; Wang, L.; Zhang, G.; Hänggi, P.; Li, B. Colloquium : Phononics: Manipulating heat flow with electronic analogs and beyond. Rev. Mod. Phys. 2012, 84, $1045-1066$.

(4) Dubi, Y.; Di Ventra, M. Colloquium : Heat flow and thermoelectricity in atomic and molecular junctions. Rev. Mod. Phys. 2011, 83, 131-155. 
(5) Yamamoto, T.; Watanabe, K. Nonequilibrium Green's Function Approach to Phonon Transport in Defective Carbon Nanotubes. Phys. Rev. Lett. 2006, 96, 255503.

(6) Wang, J.-S.; Wang, J.; Zeng, N. Nonequilibrium Green's function approach to mesoscopic thermal transport. Phys. Rev. B 2006, 74, 033408.

(7) Landauer, R. Spatial Variation of Currents and Fields Due to Localized Scatterers in Metallic Conduction. IBM Journal of Research and Development 1957, 1, 223.

(8) Zhang, M.; Lussetti, E.; de Souza, L. E. S.; MÃijller-Plathe, F. Thermal Conductivities of Molecular Liquids by Reverse Nonequilibrium Molecular Dynamics. J. Phys. Chem. B 2005, 109, 15060-15067.

(9) Lukes, J. R.; Li, D. Y.; Liang, X.-G.; Tien, C.-L. Molecular Dynamics Study of Solid Thin-Film Thermal Conductivity. J. Heat Transfer 2000, 122, 536-543.

(10) Berber, S.; Kwon, Y.-k.; Tománek, D. Unusually high thermal conductivity of carbon nanotubes. Phys. Rev. Lett. 2000, 84, 4613-4616.

(11) Ness, H.; Stella, L.; Lorenz, C. D.; Kantorovich, L. Nonequilibrium generalised Langevin equation for the calculation of heat transport properties in model 1D atomic chains coupled to two 3D thermal baths. J. Chem. Phys. 2017, 146, 164103.

(12) Ladd, A. J. C.; Moran, B.; Hoover, W. G. Lattice thermal conductivity: A comparison of molecular dynamics and anharmonic lattice dynamics. Phys. Rev. B 1986, 34, 50585064 .

(13) Volz, S. G.; Chen, G. Molecular-dynamics simulation of thermal conductivity of silicon crystals. Phys. Rev. B 2000, 61, 2651-2656.

(14) Che, J.; ÂĞä̈ß̈̈śn, T.; Deng, W.; Goddard, W. A. Thermal conductivity of diamond and related materials from molecular dynamics simulations. J. Chem. Phys. 2000, 113, 6888-6900. 
(15) Schelling, P. K.; Phillpot, S. R.; Keblinski, P. Comparison of atomic-level simulation methods for computing thermal conductivity. Physical Review B - Condensed Matter and Materials Physics 2002, 65, 1-12.

(16) McGaughey, A.; Kaviany, M. Thermal conductivity decomposition and analysis using molecular dynamics simulations. Part I. Lennard-Jones argon. Int. J. Heat Mass Transf. $2004,47,1783-1798$.

(17) McGaughey, A.; Kaviany, M. Thermal conductivity decomposition and analysis using molecular dynamics simulations: Part II. Complex silica structures. Int. J. Heat Mass Transf. 2004, 47, $1799-1816$.

(18) Chen, L.; Kumar, S. Thermal transport in graphene supported on copper. J. Appl. Phys. 2012, 112, 043502.

(19) Sellan, D. P.; Landry, E. S.; Turney, J. E.; McGaughey, A. J. H.; Amon, C. H. Size effects in molecular dynamics thermal conductivity predictions. Phys. Rev. B 2010, $81,1-10$.

(20) Baranyai, A. Heat flow studies for large temperature gradients by molecular dynamics simulation. Phys. Rev. E 1996, 54, 6911-6917.

(21) Poetzsch, R. H. H.; Bottger, H. Interplay of disorder and anharmonicity in heat conduction: Molecular-dynamics study. Phys. Rev. B 1994, 50, 757-764.

(22) Oligschleger, C. Simulation of thermal conductivity and heat transport in solids. Phys. Rev. B 1999, 59, 4125-4133.

(23) Jiang, J. W.; Lan, J.; Wang, J. S.; Li, B. Isotopic effects on the thermal conductivity of graphene nanoribbons: Localization mechanism. J. Appl. Phys. 2010, 107.

(24) Jund, P.; Jullien, R. Molecular-dynamics calculation of the thermal conductivity of vitreous silica. Phys. Rev. B 1999, 59, 13707-13711. 
(25) Müller-Plathe, F. A simple nonequilibrium molecular dynamics method for calculating the thermal conductivity. J. Chem. Phys. 1997, 106, 6082-6085.

(26) Müller-Plathe, F.; Reith, D. Cause and effect reversed in non-equilibrium molecular dynamics: An easy route to transport coefficients. Computational and Theoretical Polymer Science 1999, 9, 203-209.

(27) Bagri, A.; Kim, S. P.; Ruoff, R. S.; Shenoy, V. B. Thermal transport across twin grain boundaries in polycrystalline graphene from nonequilibrium molecular dynamics simulations. Nano Letters 2011, 11, 3917-3921.

(28) Dong, H.; Wen, B.; Melnik, R. Relative importance of grain boundaries and size effects in thermal conductivity of nanocrystalline materials. Sci. Rep. 2014, 4, 7037.

(29) Tang, S.; Kulkarni, Y. The interplay between strain and size effects on the thermal conductance of grain boundaries in graphene. Appl. Phys. Lett. 2013, 103, 213113.

(30) Si, C.; Wang, X.-D.; Fan, Z.; Feng, Z.-H.; Cao, B.-Y. Impacts of potential models on calculating the thermal conductivity of graphene using non-equilibrium molecular dynamics simulations. Int. J. Heat Mass Transf. 2017, 107, $450-460$.

(31) Goga, N.; Rzepiela, A. J.; de Vries, A. H.; Marrink, S. J.; Berendsen, H. J. C. Efficient Algorithms for Langevin and DPD Dynamics. J. Chem. Theory Comput. 2012, 8, 3637-3649.

(32) Segal, D.; Nitzan, A.; Hänggi, P. Thermal conductance through molecular wires. J. Chem. Phys. 2003, 119, 6840-6855.

(33) Klöckner, J. C.; Bürkle, M.; Cuevas, J. C.; Pauly, F. Length dependence of the thermal conductance of alkane-based single-molecule junctions: An ab initio study. Phys. Rev. B 2016, 94, 205425-1-8. 
(34) Wang, R. Y.; Segalman, R. A.; Majumdar, A. Room temperature thermal conductance of alkanedithiol self-assembled monolayers. Appl. Phys. Lett. 2006, 89, 173113.

(35) Meier, T.; Menges, F.; Nirmalraj, P.; Hölscher, H.; Riel, H.; Gotsmann, B. LengthDependent Thermal Transport along Molecular Chains. Phys. Rev. Lett. 2014, 113, 060801 .

(36) Majumdar, S.; Sierra-Suarez, J. A.; Schiffres, S. N.; Ong, W.-L.; Higgs, C. F.; McGaughey, A. J. H.; Malen, J. A. Vibrational Mismatch of Metal Leads Controls Thermal Conductance of Self-Assembled Monolayer Junctions. Nano Letters 2015, 15, 29852991.

(37) Cui, L.; Hur, S.; Akbar, Z. A.; Klöckner, J. C.; Jeong, W.; Pauly, F.; Jang, S.-Y.; Reddy, P.; Meyhofer, E. Thermal conductance of single-molecule junctions. Nature 2019 ,

(38) Crljen, Ž.; Baranović, G. Unusual conductance of polyyne-based molecular wires. Phys. Rev. Lett. 2007, 98, 1-4.

(39) Garner, M. H.; Bro-Jørgensen, W.; Pedersen, P. D.; Solomon, G. C. Reverse BondLength Alternation in Cumulenes: Candidates for Increasing Electronic Transmission with Length. J. Phys. Chem. C 2018, acs.jpcc.8b05661.

(40) Torii, D.; Nakano, T.; Ohara, T. Contribution of inter- and intramolecular energy transfers to heat conduction in liquids. J. Chem. Phys. 2008, 128.

(41) Pronk, S.; PÃąll, S.; Schulz, R.; Larsson, P.; Bjelkmar, P.; Apostolov, R.; Shirts, M. R.; Smith, J. C.; Kasson, P. M.; van der Spoel, D.; Hess, B.; Lindahl, E. GROMACS 4.5: a high-throughput and highly parallel open source molecular simulation toolkit. Bioinformatics 2013, 29, 845-854. 
(42) O'Boyle, N. M.; Banck, M.; James, C. A.; Morley, C.; Vandermeersch, T.; Hutchison, G. R. Open Babel: An open chemical toolbox. J. Cheminformatics 2011, 3, 33.

(43) Hanwell, M. D.; Curtis, D. E.; Lonie, D. C.; Vandermeersch, T.; Zurek, E.; Hutchison, G. R. Avogadro: an advanced semantic chemical editor, visualization, and analysis platform. Journal of Cheminformatics 2012, 4, 17.

(44) Rappe, A. K.; Casewit, C. J.; Colwell, K. S.; Goddard, W. A.; Skiff, W. M. UFF, a full periodic table force field for molecular mechanics and molecular dynamics simulations. Journal of the American Chemical Society 1992, 114, 10024-10035.

(45) Zhang, Y.; Barnes, G. L.; Yan, T.; Hase, W. L. Model non-equilibrium molecular dynamics simulations of heat transfer from a hot gold surface to an alkylthiolate selfassembled monolayer. Phys. Chem. Chem. Phys. 2010, 12, 4435-4445.

(46) Carles, R.; Benzo, P.; Pécassou, B.; Bonafos, C. Vibrational density of states and thermodynamics at the nanoscale: the 3D-2D transition in gold nanostructures. Sci. Rep. 2016, 6, 39164.

(47) Altman, D. G.; Bland, J. M. Standard deviations and standard errors. BMJ (Clinical research ed.) 2005, 331, 903. 


\section{Graphical TOC Entry}

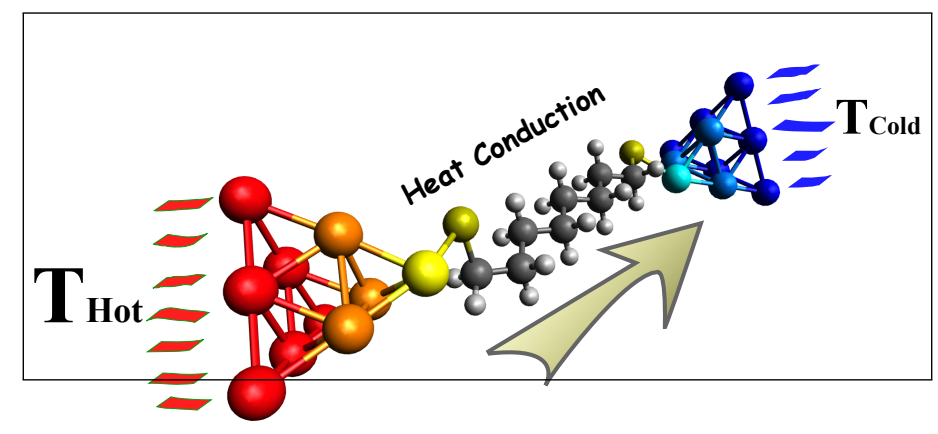

Figure 9 


\title{
Supporting Information for Stochastic
}

\section{Simulation of Nonequilibrium Heat}

\section{Conduction in Extended Molecule Junctions}

\author{
Inon Sharony, ${ }^{\dagger}$ Renai Chen, ${ }^{\ddagger}$ and Abraham Nitzan*,†, \\ $\dagger$ †chool of Chemistry, Tel Aviv University, Tel Aviv 69978, Israel \\ $\ddagger$ Department of Chemistry, University of Pennsylvania, Philadelphia, PA 19104, USA \\ E-mail: anitzan@sas.upenn.edu
}

\section{S1. Details on molecular configuration preparation and MD simulation procedures}

\begin{abstract}
There are mainly three phases in using our customized GROMACS ${ }^{1}$ package to conduct simulations on heat conduction in molecular junctions, including both classical MD and quantum Landauer transport (as illustrated in figure S1):
\end{abstract}




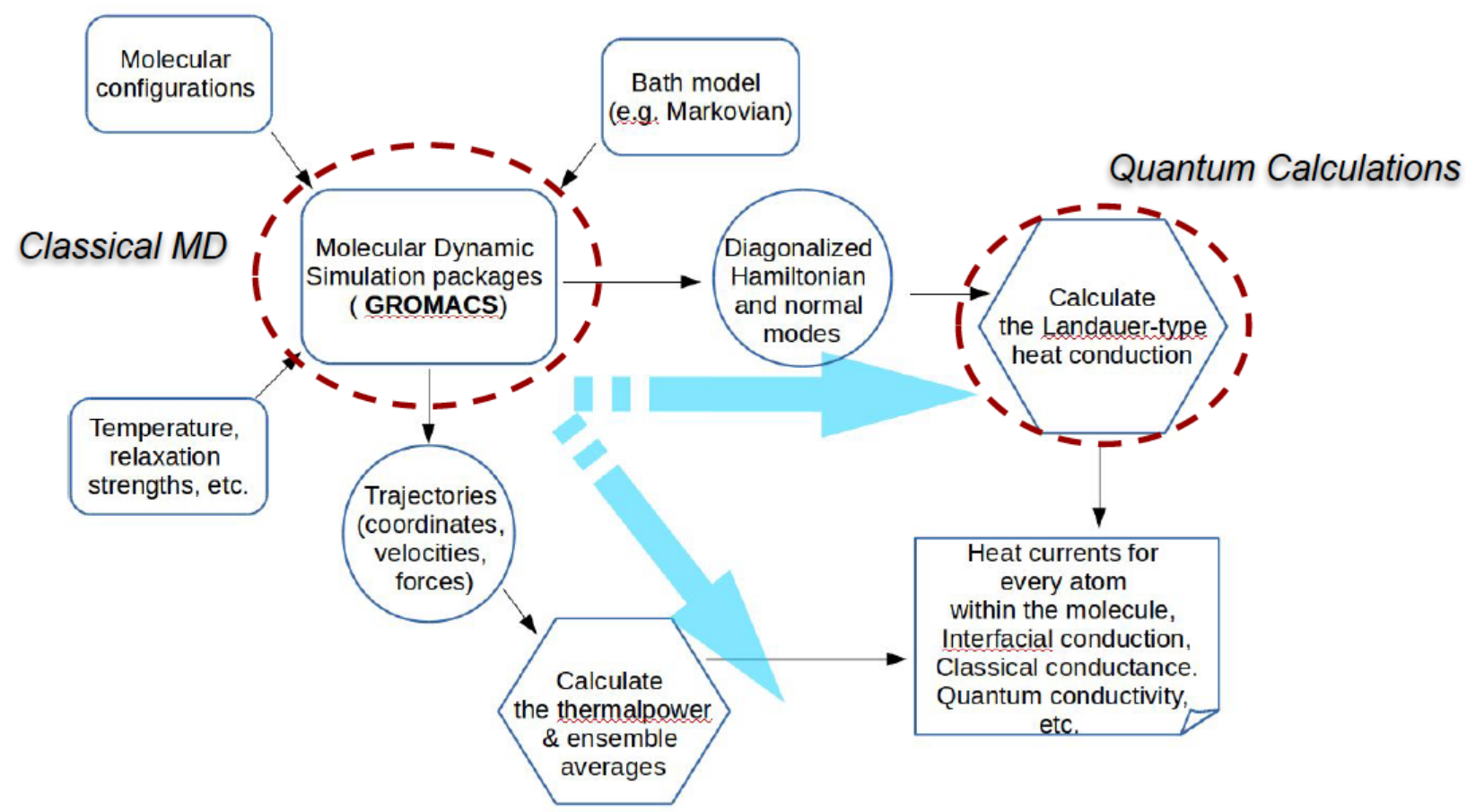

Figure S1: Molecular heat conduction MD simulation procedure and implementation diagram, with Landauer-type quantum calculations included.

(I) Configuration preparation. The open-source graphic cross-platform molecule editor, Avogardro, ${ }^{2}$ is used to design the initial molecular topologies as one wishes (e.g. alkanedithiols with three layers of gold substrates). Then these configurations are energy minimized with Avogardro's native utility, and saved as $p d b$ formats, which are eventually transformed into GROMACS's recognizable input files (gro and top) using Open Babel ${ }^{3}$ with Uinversal Force Field (UFF $)^{4}$ implemented as molecular force field parameters.

(II) Production runs. Within GROMACS the molecualr configurations are further energy minimized to ensure stable structure for subsequent dynamical runs and normal mode analysis. For MD production runs, the systems are first equilibrated to the average temperatures of the baths (usually takes a few hundred pecoseconds), and then with a single long trajectory (a few nanoseconds) relaxed to nonequilibrium steady state. From the tail of this trajectory, thousands of parallel at-steady-state trajectories are launched get large enough statisical ensembles. At the end of this phase, as outputs, normal modes, steady-state trajectories, and inter-atomic forces are collected. 
(III) Post-data processing. The heat currents are calculated according to the equation in the Main text using velocities and inter-atomic forces sampled at the end of the last step. The Landauer-type currents are calcualted through a different channel, by using the formalism described in the last section of this SI. The resultant quantities (e.g. currents, local temperatures, conductance, etc.) are averaged (with error estimation) and plotted for illustration purposes.

\section{S2. Supplemental MD simulation results}

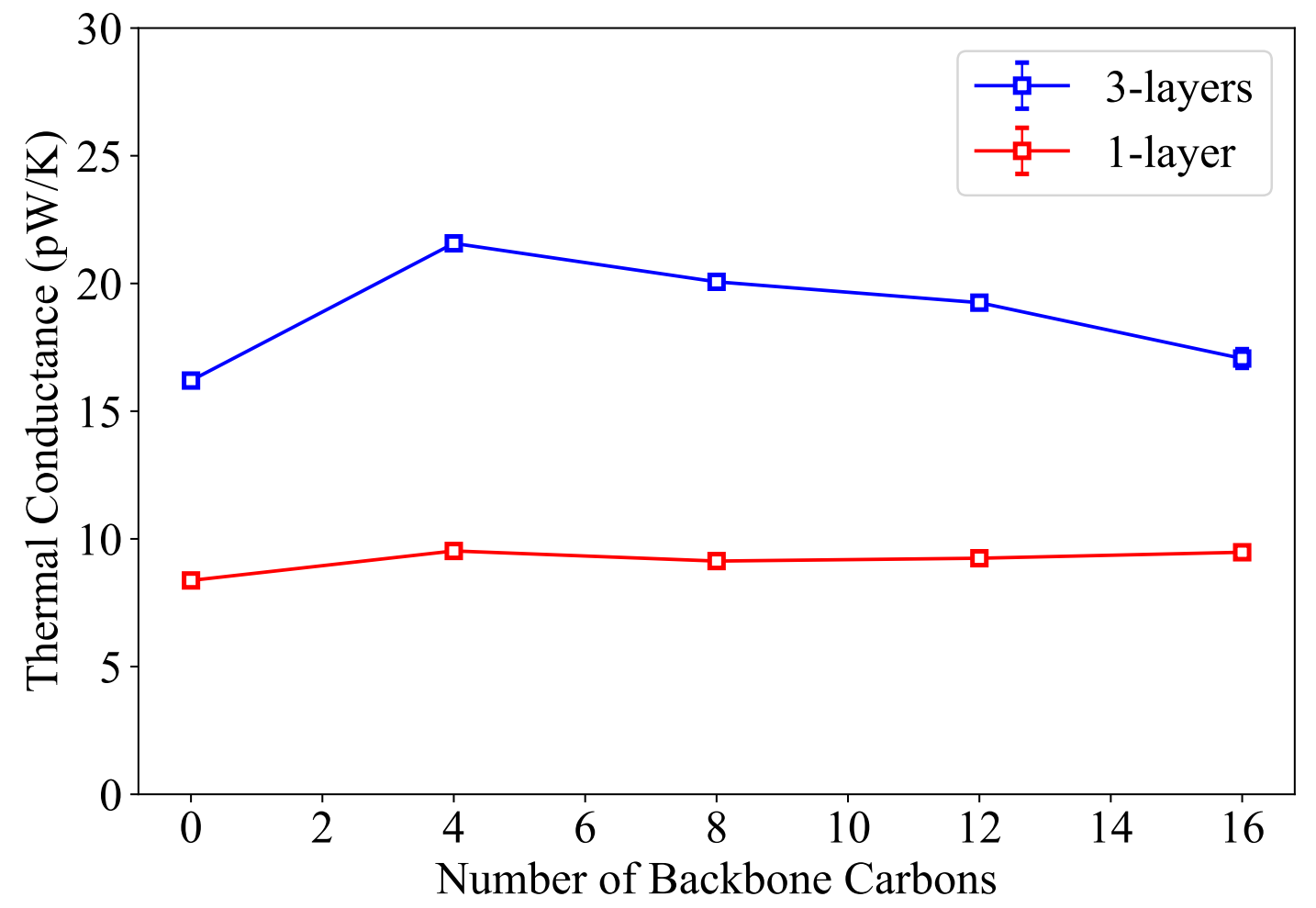

Figure S2: Heat conductance simulated for alkanes of various lengths, under explicit baths of different number of explicit Gold layers. The bath temperatures are set at 300K to 350K. The bars shown in the figure are the standard errors. 


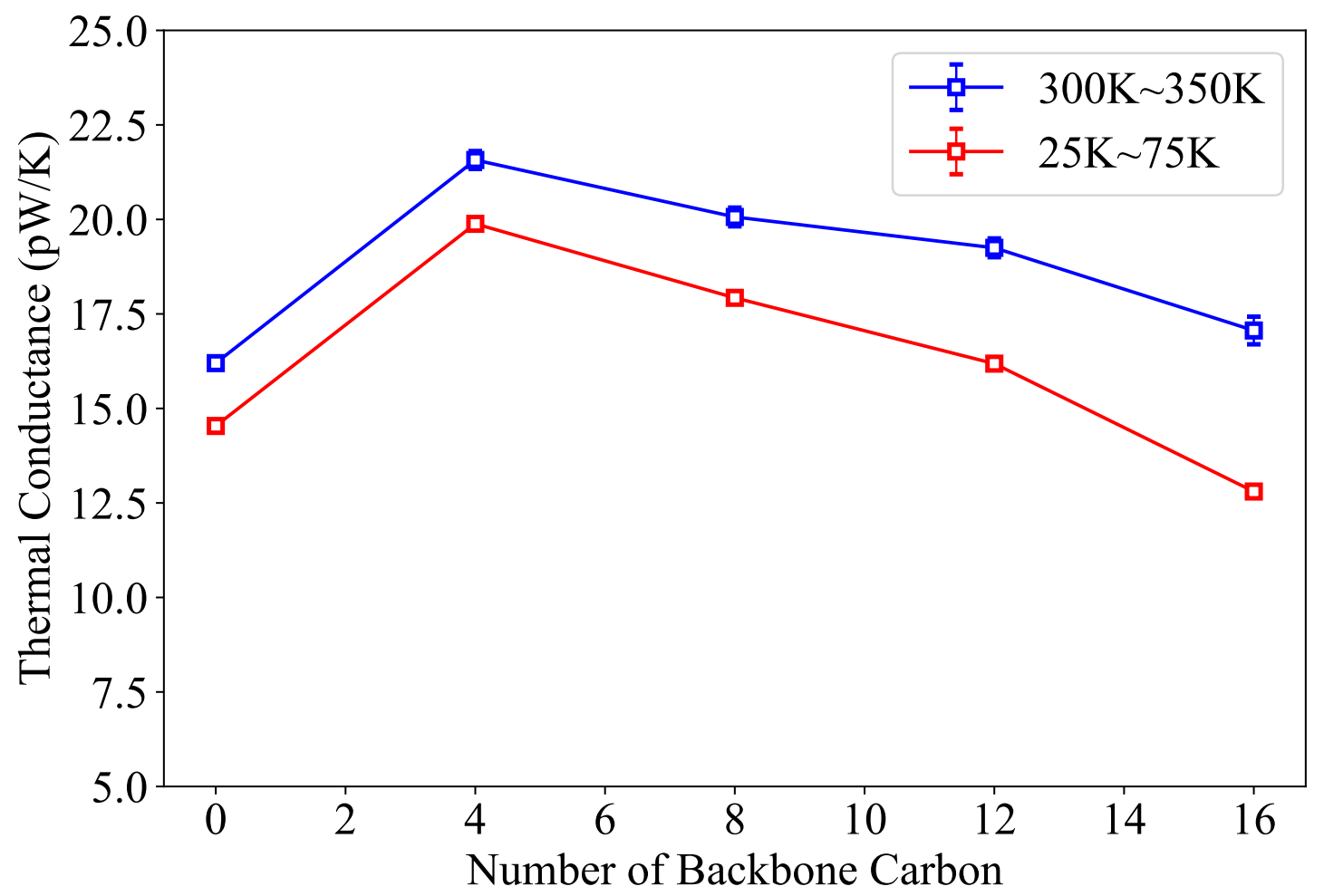

Figure S3: Comparison of low temperature conductance with room temperature conductance for different length of alkanedithiols with 3-layers of explicit gold bulks, from GROMACS MD simulations.

\section{S3. Landauer-type calculations for hydrocarbon heat con- duction}

\section{Landauer formalism}

When only the harmonic part of the system-bath interactions are taken into account, the (phononic) heat current can be expressed by the Landauer formula,

$$
J=\frac{\hbar}{2 \pi} \int_{0}^{\infty} \mathcal{T}(\omega)\left[f\left(\omega, T_{L}\right)-f\left(\omega, T_{R}\right)\right] \omega d \omega
$$


which has been derived in various theoretical approaches and employed in different systems. ${ }^{5-11}$ Here $f$ function is the Bose-Einstein distribution function which depends the temperature of the bath, $f(\omega, T)=\left(e^{\hbar \omega / k_{B} T}-1\right)^{-1}$, and $\mathcal{T}$ is the transmission probability.

In the Linear Response regime, the thermal conductance is taken as the derivative of the heat current with respect to the temperature,

$$
\kappa=\frac{\hbar}{2 \pi} \int_{0}^{\infty} \mathcal{T}(\omega) \frac{\partial f(\omega, T)}{\partial T} \omega d \omega
$$

The transmission function is often expressed using the Meir-Wingreen formula ${ }^{12}$

$$
\mathcal{T}(\omega)=\operatorname{Tr}\left[\boldsymbol{G}_{S}^{r}(\omega) \boldsymbol{\Gamma}_{L}(\omega) \boldsymbol{G}_{S}^{a}(\omega) \boldsymbol{\Gamma}_{R}(\omega)\right]
$$

in which $\boldsymbol{G}_{S}^{a}=\left[\boldsymbol{G}_{S}^{r}\right]^{\dagger}$, are the advanced \& retarded Green's functions of the system and can be written as, ${ }^{6}$

$$
\boldsymbol{G}_{S}^{r / a}(\omega)=\left[\omega^{2} \boldsymbol{M}-\boldsymbol{D}-\left(\boldsymbol{\Sigma}_{L}^{r / a}+\boldsymbol{\Sigma}_{R}^{r / a}\right)\right]^{-1}
$$

most easily understood in the basis of the atomic coordinates, $\boldsymbol{D}$ is the dynamical matrix (or Hessian) whose elements are the second derivatives of the total energy with respect to the atomic coordinates, $\boldsymbol{M}$ is the (diagonal) matrix of atomic masses, and $\boldsymbol{\Sigma}_{K}^{r / a}$ is the retarded (or advanced, respectively) self-energy of the $K$ bath $(K \in L, R)$.

$\Gamma_{L}$ and $\Gamma_{R}$ are the spectral function matrices of the baths, reflecting the coupling strengths of the systems to the leads.

$$
\Gamma_{L / R}(\omega)=i\left[\Sigma_{L / R}^{r}(\omega)-\Sigma_{L / R}^{a}(\omega)\right]
$$

When spectral density is taken as Ohmic, ${ }^{5,7}$ the self-energies are defined as $\left[\boldsymbol{\Sigma}_{L}^{r}\right]_{i j} \equiv$ $-i \omega \gamma_{L}(\omega) \delta_{i j}$ for all atomic indexes $i$ (and $j$ ) which correspond to coordinates of atoms at the 
left-most layer of bulk, and directly coupled to the left heat reservoir, which is characterised by a dissipation coefficient $\gamma_{L}(\omega)$. Correspondingly for the right bath. Also, taking both baths to be white, $\gamma_{L}(\omega)$ and $\gamma_{R}(\omega)$ are constant.

\section{Methodology}

The main ingredient needed for the use of the Landauer formula in molecular systems is to reliably obtain the dynamical force matrix $\boldsymbol{D}$. The GROMACS ${ }^{1}$ software package provides utilities which construct the Hessian matrix of the system under study. Furthermore, it has the ability to diagonalize the obtained Hessian, providing the eigenfrequencies and eigenvectors (normal modes) of the system. 


\section{Results}

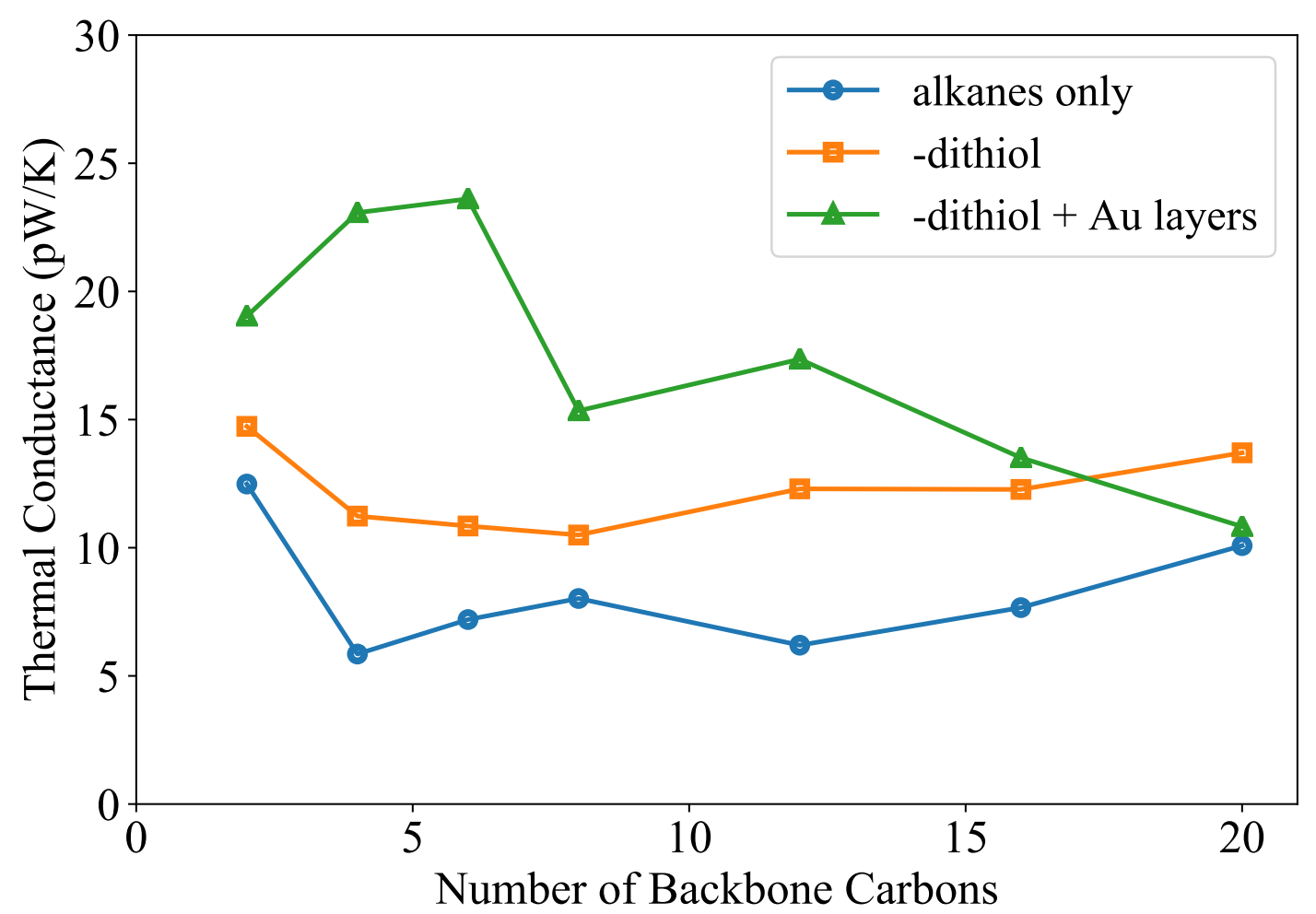

Figure S4: Length-dependent thermal conductance, calculated through the Landauer formula. The exterior-most layers of explicit bulk are coupled to white noise baths. The conductance denotes ratio between heat current and temperature bias, with left and right baths at $300 \mathrm{~K}$ and $350 \mathrm{~K}$ respectively. There are three Gold layers, in accordance with the MD simulations.

By adding layers to different alkane molecules(Figure S4), we find

1. The presence of thiol groups enhances alkane conductance.

2. The trend of conductance increase and eventual decrease (as a function of chain length), as reported in ab-initio calculations of similar systems, ${ }^{11}$ appears only when including the layers of Gold leads, explicitly.

To see how different molecular vibrational modes line up with the bath spectral density, the Normal Mode Analysis (NMA) of various molecules is performed (results shown in Figure S5). 


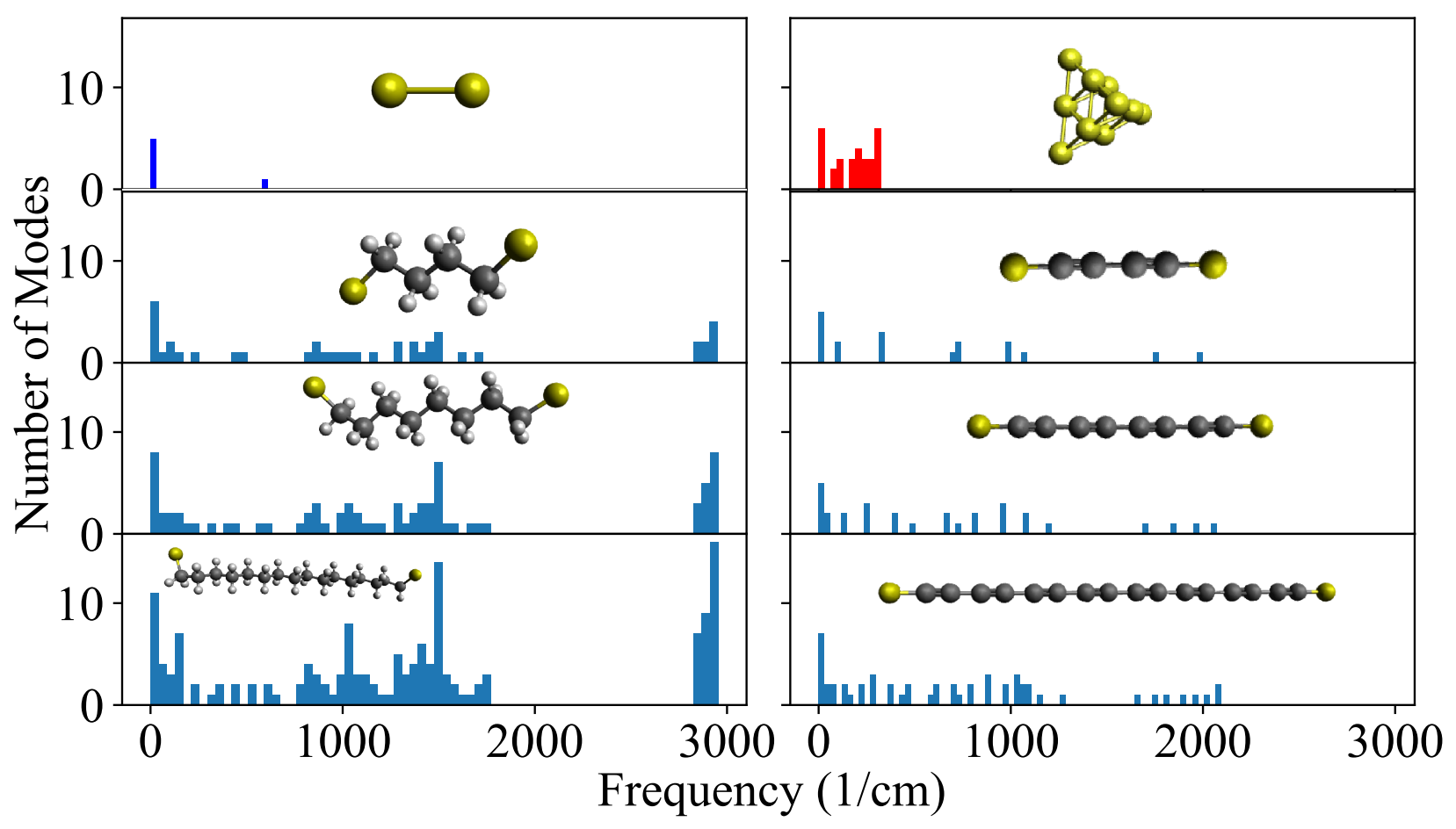

Figure S5: Histograms of the Normal Mode Analysis of some representative molecules. The $\mathrm{x}$-axes are the histogram bins (frequencies in wavenumbers), and the y-axes are the count of modes with frequency in the bin. The corresponding molecular topologies are drawn within each panel. Note that the first and last atom in each molecule are Sulfur. The first row shows the normal mode histogram for disulfur and a three layer gold cluster (left and right columns, respectively). The following rows depict alkanes (left) and polyyens (right) with 4 , 8, and 16 Carbon units, respectively.

While the molecules have some modes up to a thousand wavenumbers, the significant band of bulk phonons exists between a few tens and a few hundred wavenumbers, as seen in gold bulk spetrum density figure in the main text. The high-frequency molecular vibrational modes, therefore, do not overlap with the bulk modes. The prominent peaks of molecular vibrations at lower frequencies are due to rigid-body Center of Mass Motion, or such Degrees of Freedom as they are hybridized with vibrations. Other factors which could be affecting the overall heat conduction include, normal mode localization, ${ }^{8}$ system-bath coupling, transmission coefficients in the picture of coherent transport. ${ }^{11}$ The relevant investigations will require not only analysis of energy flows within the molecules and between molecules and baths, but also in frequency space, and detailed comparisons to Landauer-type conductivity calculations. 
We compare the localization properties of the normal modes for the molecules of different lengths and saturations. Assuming the transformation coefficients for changing the local atomic basis to normal mode basis to be $C_{n_{i}, \alpha}$, then a localization factor (also called participation index) for each mode can be defined as: ${ }^{8}$

$$
P_{\alpha}(n)=\sum_{n_{i}}\left|C_{n_{i}, \alpha}\right|^{2}
$$

where $\mathrm{n}$ is the index of atoms in the molecule, $\alpha$ denote the modes, $n_{i}$ is the degrees of freedom which goes from 1 to 3 for each $n$. To see the localization on carbon based backbone clusters, the factor can further be summed up over nearby hydrogens.

$$
P_{\alpha}\left(n_{B}\right)=\sum_{n=1}^{n_{H}} P_{\alpha}(n),
$$

where $n_{H}$ denotes the bonded hydrogens to backbone atom $n_{B}$. 

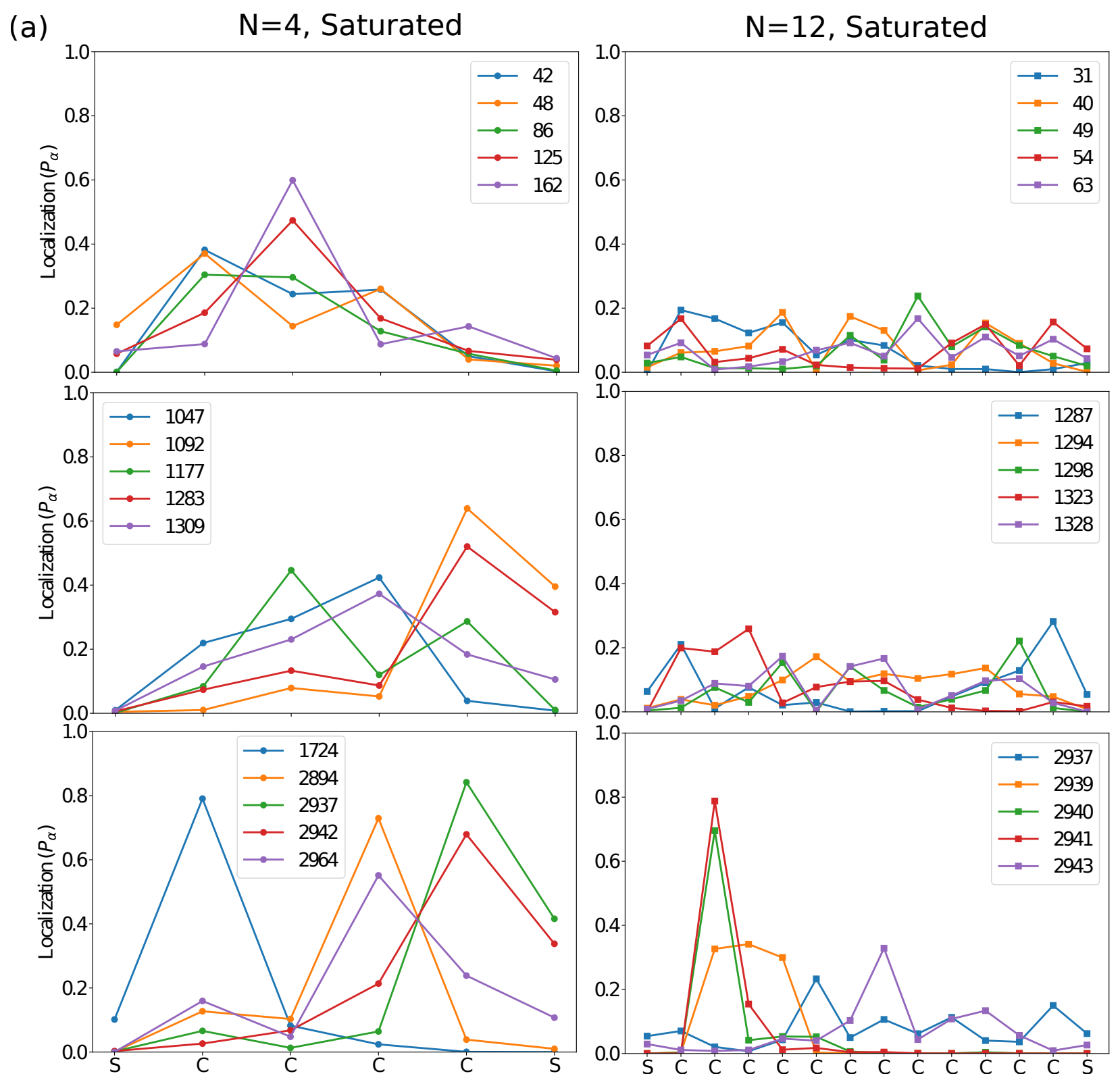
(b)
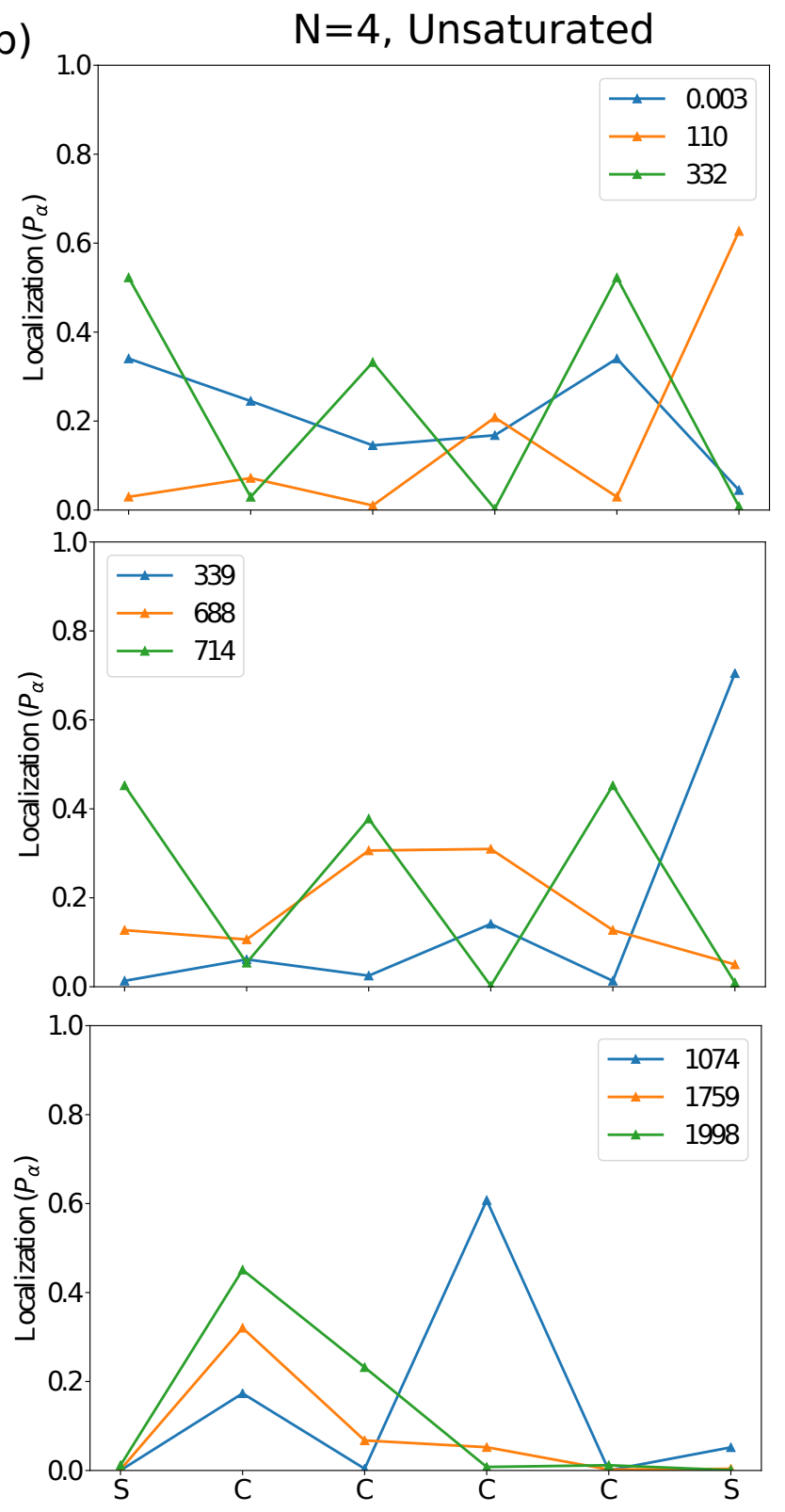
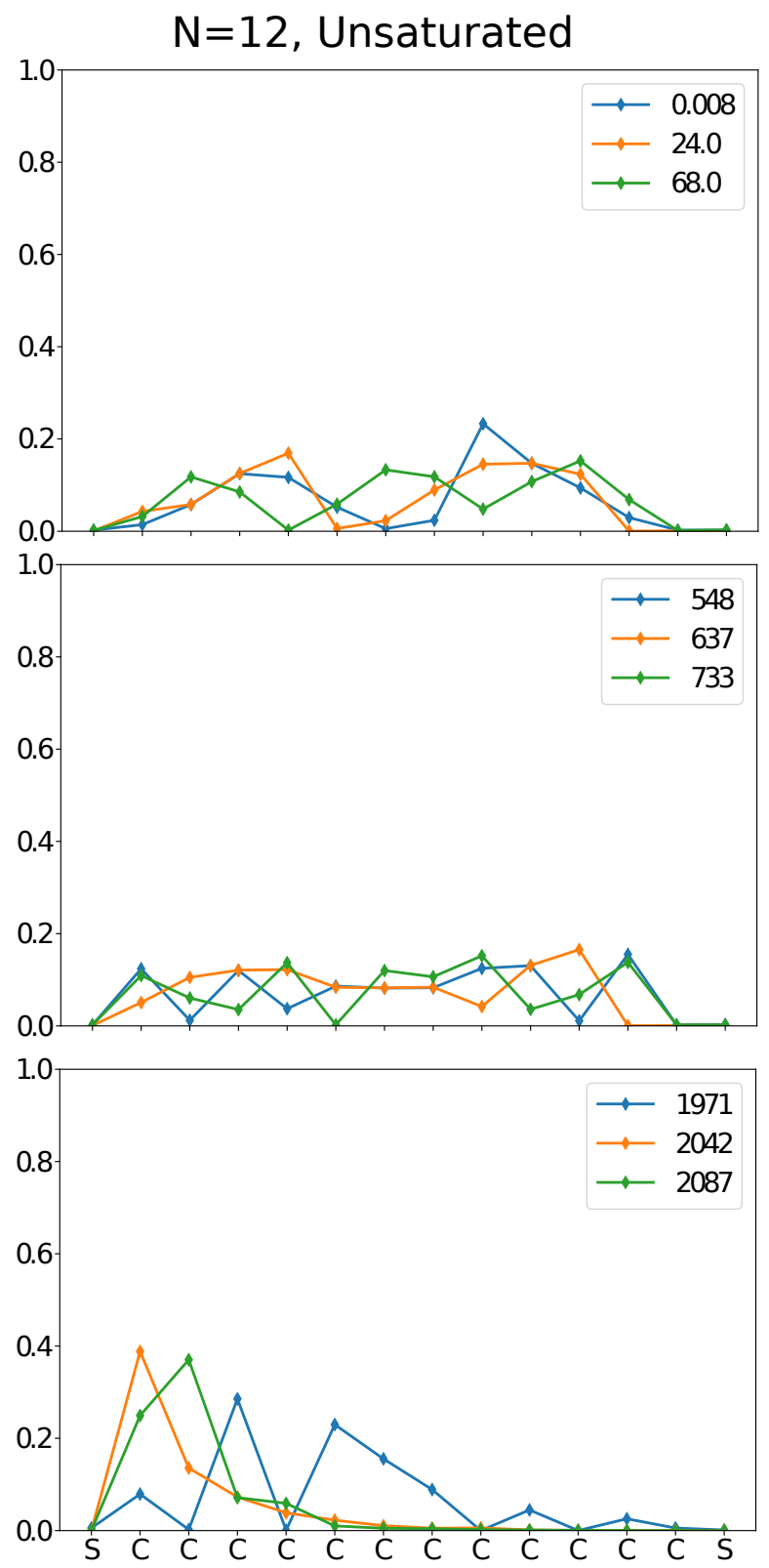

Figure S6: Localization coefficients for different hydrocarbon molecules. $N$ is the number of backbone carbon atoms, and all molecules are capped with thiol groups. "Saturated" refers to alkanedithiols(upper six panels labeled as (a)), while "Unsaturated" refers to conjugated carbon chains with alternating single and triple bond (Polyynes)(lower six pannels labeled as (b)). The numbers in the legends are the corresponding frequencies (unit in $\mathrm{cm}^{-1}$, ranging from low, medium to high) of the selected normal modes of the hydrocarbon chain molecules.

From figure S6, the high frequency modes $\left(\sim 3000 \mathrm{~cm}^{-1}\right.$ for alkanes and $\sim 2000 \mathrm{~cm}^{-1}$ for polyynes) seem more spatially localized than low (less than $\left.100 \mathrm{~cm}^{-1}\right)$ and medium $(\sim$ $1200 \mathrm{~cm}^{-1}$ for alkanes and $\sim 600 \mathrm{~cm}^{-1}$ for polyynes) frequency modes, which might indicate 
that those high frequency modes are less conductive even when the baths contain high frequency phonons. Nonetheless, one has to be careful not to ignore the couplings among molecular modes and between system and baths as well.
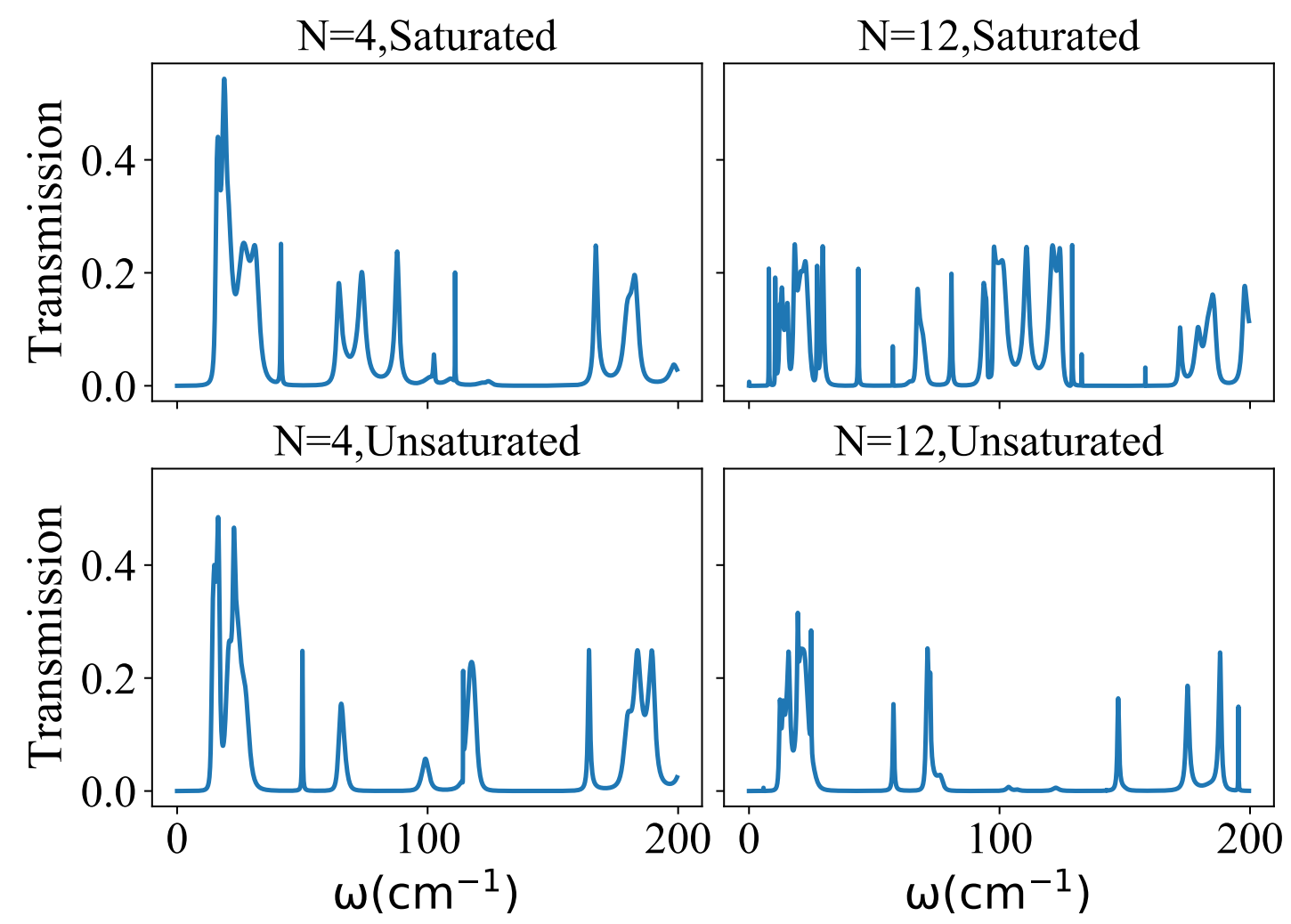

Figure S7: The transmission probabilities $\mathcal{T}(\omega)$ for different hydrocarbon molecules. $N$ is the number of backbone carbon atoms, and all molecules are capped with thiol groups. "Saturated" refers to alkanedithiols, while "Unsaturated" refers to conjugated carbon chains with alternating single and triple bond (Polyynes). Each molecule is adsorbed on a gold surface modeled using 3 explicit bulk layers.

As an important component in Landuaer's description, transmission coefficients may become more a direct indicator of heat conduction compared to normal mode density and localization. The comparison of transmission between short and long chains, of both saturated and unsaturated hydrocarbons (Figure S7), provides insights into the contribution of different vibrational modes to the overall heat conduction. Based on these results, one may make a qualitative observation that the short saturated hydrocarbons are the most conducting, while long unsaturated hydrocarbons are less conductive. 


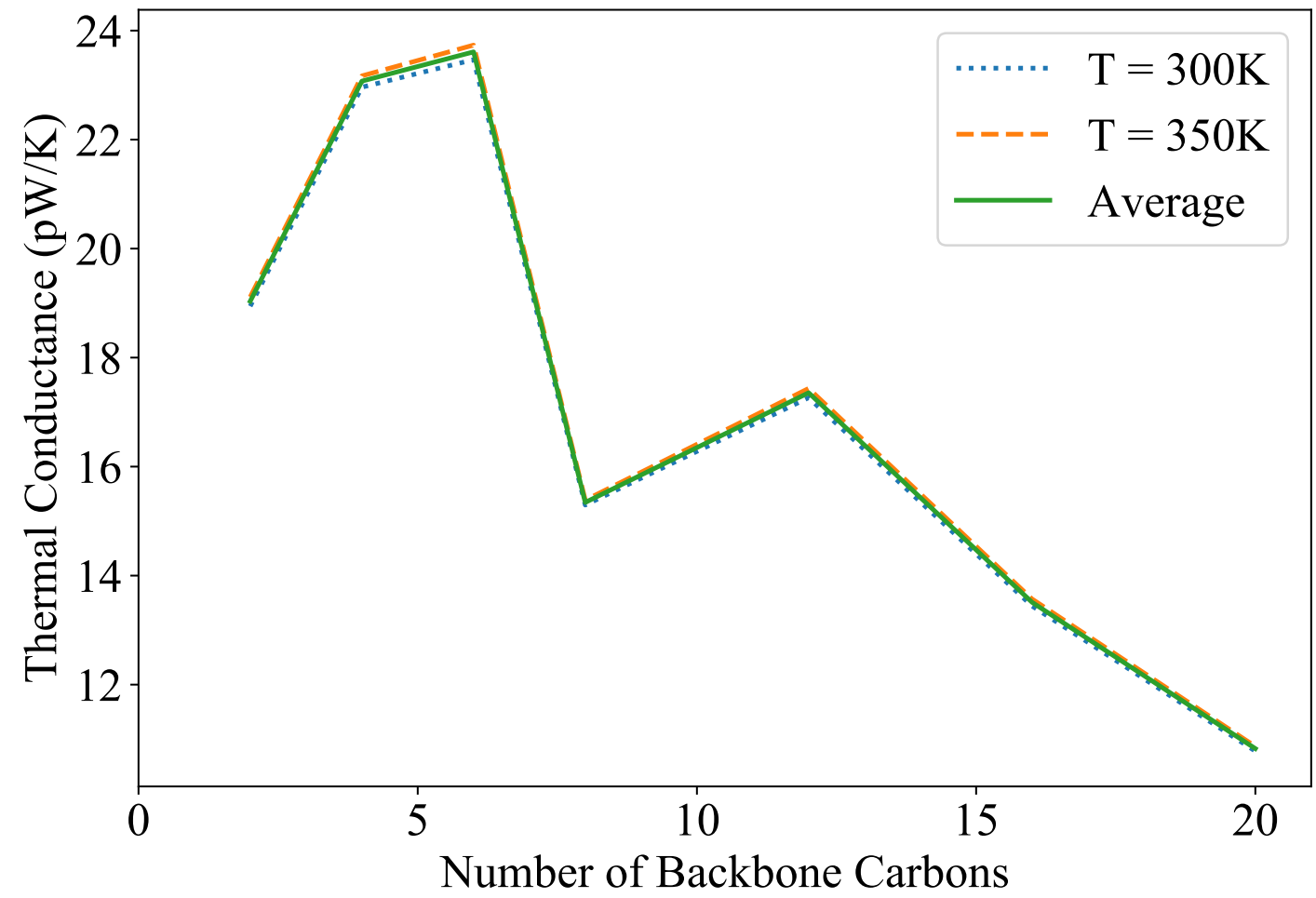

Figure S8: Comparison between derivative conductance and finite-bias conductance. For the finite-bias case, bath temperatures of $300 \mathrm{~K}$ and $350 \mathrm{~K}$ are taken (i.e. bias of $50 \mathrm{~K}$ ). This is compared with two derivative conductance calculations: Around a temperature of $300 \mathrm{~K}$ and of $350 \mathrm{~K}$.

We would now like to see how the choices of different conductance expressions (using the derivative form or the finite-bias form) influence the results. It turns out that the influence is negligible across a range of alkane chain lengths (Figure S8). The conductance at 350K is slightly higher and 300K slightly lower, but the difference between either of them and the finite-bias result is significantly smaller than the values of the relevant conductances themselves. 


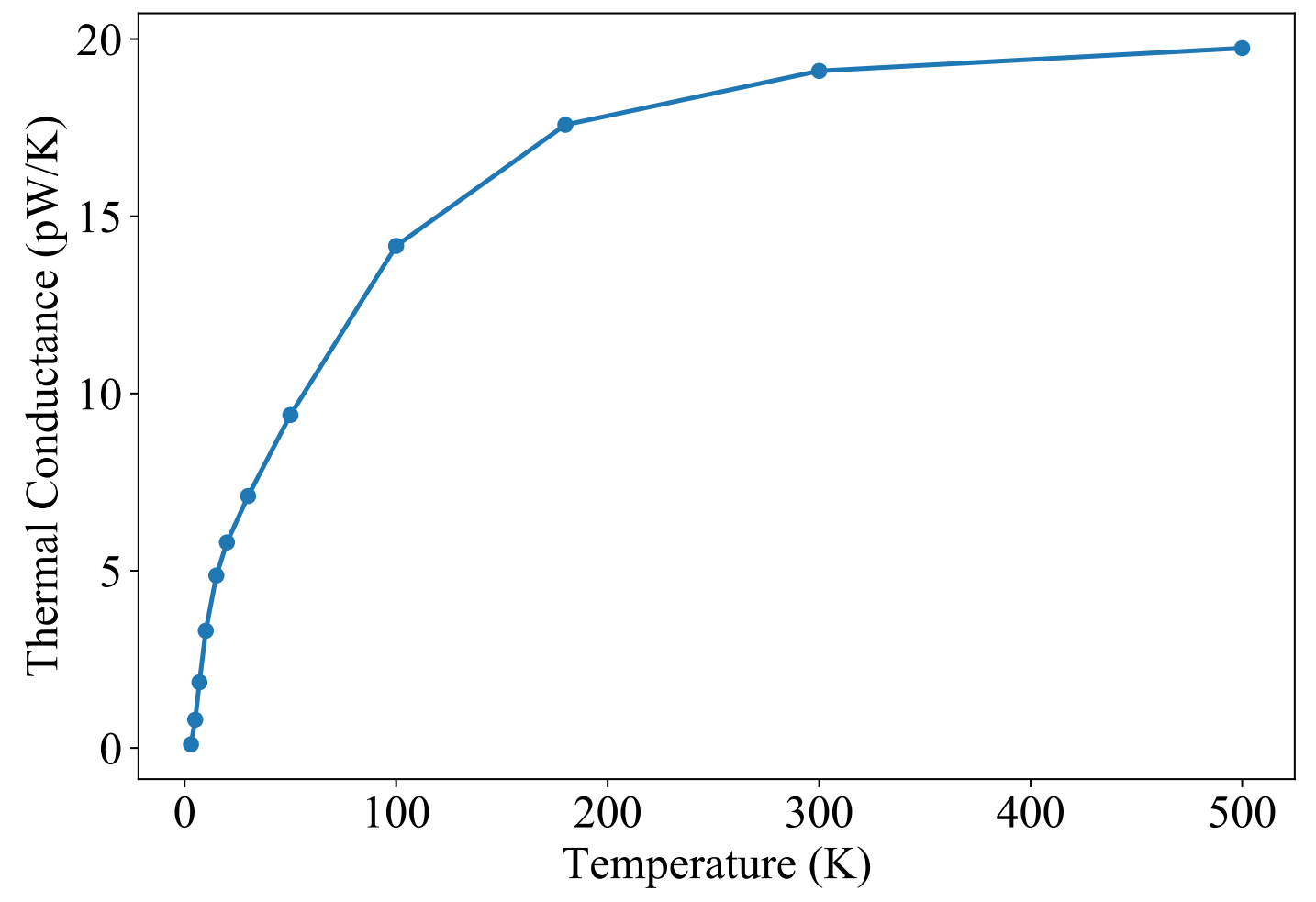

Figure S9: Temperature dependence of the derivative Landuaer thermal conductance, for butanedithiol $\left(\mathrm{HS}\left(\mathrm{CH}_{2}\right)_{10} \mathrm{SH}\right)$ adsorbed on a substrate modelled by 3 layers of explicit Gold atoms.

Figure S9 shows the temperature dependence of the heat conductance of butanedithiol. Conductance increases at a higher rate until 100K, and then starts to plateau as temperature increases further. This trend agrees with results reported for an identical alkane. ${ }^{11}$

The comparison of conductance between high (300K to $350 \mathrm{~K})$ and low (25K to $75 \mathrm{~K})$ (Figure S10) temperatures from Landauer calculations gives us a sense of the importance of the temperature factor plays in the molecular heat conduction simulations. From this perspective, the classical MD simulations (Figure S3 is not a reliable reference in the low temperature limit. The characteristic energy of phonons will be much lower, and thus the conductance at low temperature. The classical simulation does not show any major difference when comparing the high temperature profiles (Figure S3). Therefore, we may propose a combination of probing heat conduction with classical MD at high temperature (where scattering might be 


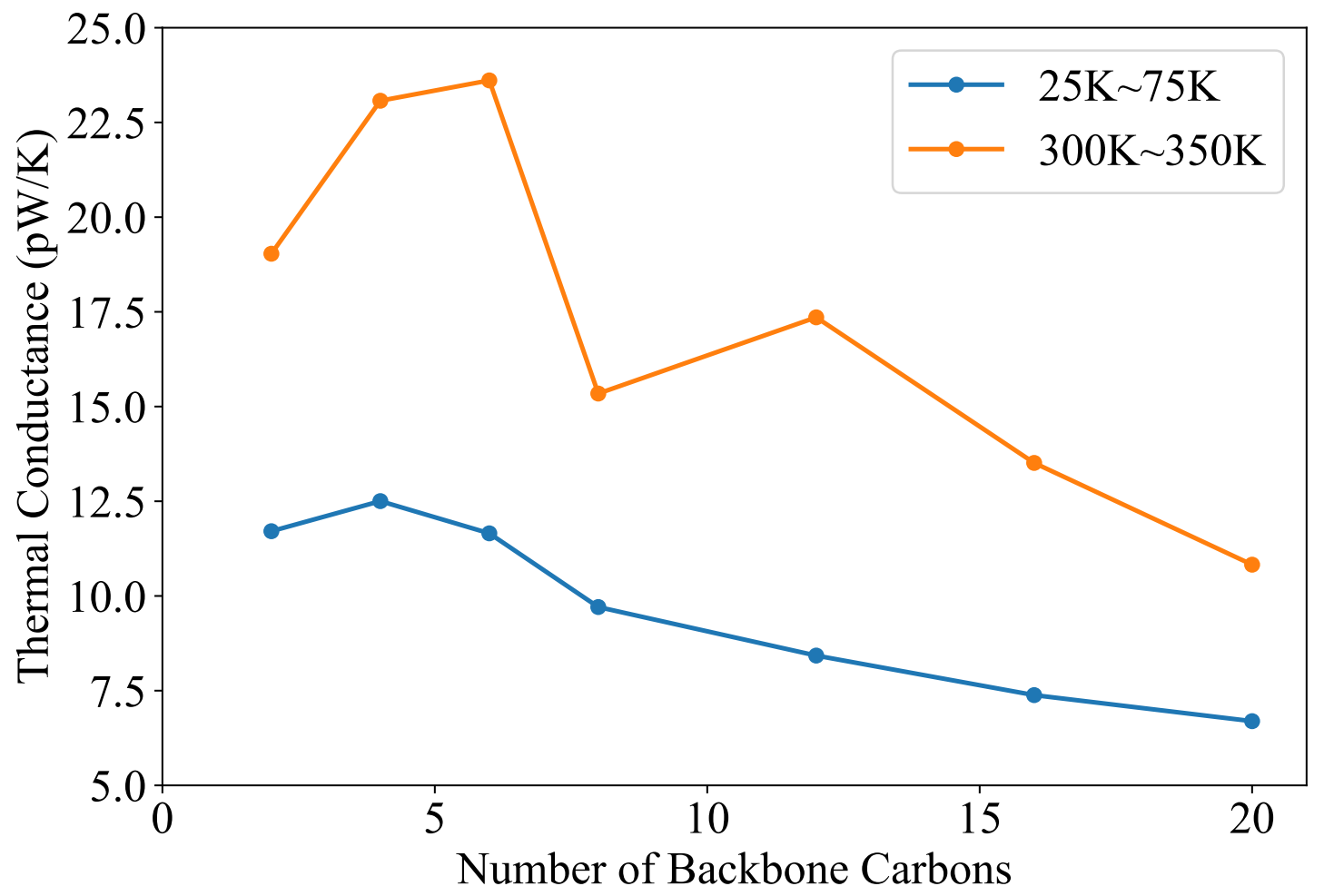

Figure S10: Comparison of Landauer conductance at low vs. room temperatures (temperature bias the same in both cases) for alkanedithiols of different lengths, with 3-layers of explicit gold bulks. 
more important), and with quantum Landauer's approach at low temperature limit (where the harmonic interaction dominates). These benefits are all included in our newly developed simulation package presented here.

\section{References}

(1) Pronk, S.; PÃąll, S.; Schulz, R.; Larsson, P.; Bjelkmar, P.; Apostolov, R.; Shirts, M. R.; Smith, J. C.; Kasson, P. M.; van der Spoel, D.; Hess, B.; Lindahl, E. GROMACS 4.5: a high-throughput and highly parallel open source molecular simulation toolkit. Bioinformatics 2013, 29, 845-854.

(2) Hanwell, M. D.; Curtis, D. E.; Lonie, D. C.; Vandermeersch, T.; Zurek, E.; Hutchison, G. R. Avogadro: an advanced semantic chemical editor, visualization, and analysis platform. Journal of Cheminformatics 2012, 4, 17.

(3) O’Boyle, N. M.; Banck, M.; James, C. A.; Morley, C.; Vandermeersch, T.; Hutchison, G. R. Open Babel: An open chemical toolbox. J. Cheminformatics 2011, 3, 33.

(4) Rappe, A. K.; Casewit, C. J.; Colwell, K. S.; Goddard, W. A.; Skiff, W. M. UFF, a full periodic table force field for molecular mechanics and molecular dynamics simulations. Journal of the American Chemical Society 1992, 114, 10024-10035.

(5) Segal, D.; Agarwalla, B. K. Vibrational Heat Transport in Molecular Junctions. Annu. Rev. Phys. Chem. 2016, 67, 185-209.

(6) Dhar, A.; Roy, D. Heat Transport in Harmonic Lattices. J. Stat. Phys. 2006, 125, 801-820.

(7) Dhar, A. Heat transport in low-dimensional systems. Adv. Phys. 2008, 57, 457-537.

(8) Segal, D.; Nitzan, A.; Hänggi, P. Thermal conductance through molecular wires. J. Chem. Phys. 2003, 119, 6840-6855. 
(9) Wang, J.-S.; Wang, J.; Zeng, N. Nonequilibrium Green's function approach to mesoscopic thermal transport. Phys. Rev. B 2006, 74, 033408.

(10) Yamamoto, T.; Watanabe, K. Nonequilibrium Green's Function Approach to Phonon Transport in Defective Carbon Nanotubes. Phys. Rev. Lett. 2006, 96, 255503.

(11) Klöckner, J. C.; Bürkle, M.; Cuevas, J. C.; Pauly, F. Length dependence of the thermal conductance of alkane-based single-molecule junctions: An ab initio study. Phys. Rev. B 2016, 94, 205425-1-8.

(12) Meir, Y.; Wingreen, N. S. Landauer formula for the current through an interacting electron region. Phys. Rev. Lett. 1992, 68, 2512-2515. 